\title{
AN INVESTIGATION INTO THE USE OF POLYMER BOUND BORONIC ACID FOR GLUCOSE DETECTION IN PAPER-BASED MICROFLUIDIC DEVICES
}

\author{
A Thesis \\ presented to \\ the Faculty of California Polytechnic State University, \\ San Luis Obispo \\ In Partial Fulfillment \\ of the Requirements for the Degree \\ Master of Science in Polymers and Coating Science
}

by

Spencer A. Schultz

June 2016 
(C) 2016

Spencer A. Schultz

ALL RIGHTS RESERVED 
TITLE:

DATE SUBMITTED:

COMMITTEE CHAIR: Andres W. Martinez, Ph.D.

COMMITTEE MEMBER: $\quad$ Raymond H. Fernando, Ph.D.

Professor and Endowed Chair of Polymers and Coating Science

COMMITTEE MEMBER: John P. Hagen, Ph.D.

Professor of Chemistry and Biochemistry 


\begin{abstract}
An Investigation into the Use of Polymer Bound Boronic Acid for Glucose Detection in Paper-Based Microfluidic Devices

Spencer A. Schultz

Paper Based Microfluidic Devices (microPADs) are a new platform for point-ofcare diagnostic assays for use in resource-limited settings. These devices rely typically on enzymatic assays to produce their results, which makes them susceptible to degradation when exposed to extreme environmental conditions such as high temperature. In order to overcome this limitation, this research project focused on investigating the use of polymers instead of enzymes to detect analytes on microPADs.
\end{abstract}

Polymer-bound boronic acid, a glucose and $\mathrm{pH}$ sensitive polymer, was incorporated into microPADs in order to develop a chronometric, paper-based glucose assay. The polymer was tested with both lateral and vertical flow microPADs made from three different types of paper, and several different methods of incorporating the polymer into the devices were also explored. While some devices appeared to show a trend in signal versus concentration of glucose, none of the results were statistically significant due to the large standard deviations in the signal. Upon further analysis of the results, the overall conclusion was that the devices were not sensitive enough to detect glucose in the range of concentrations that would be practical for clinical diagnostic applications. 


\section{ACKNOWLEDGMENTS}

My time here at Cal Poly has been a great learning experience even if some of my lessons were learned the hard way and has done so much to prepare me for both further graduate school and for a scientific career. In addition, Cal Poly broadened my scientific knowledge and allowed me experiences that I would never have dreamed of. Finally, Cal Poly allowed me to stay in my home area while working on my Master of Science degree and has allowed me through generous help from both the institution and the graduate program to graduate without any student debt, which is not something that many college and graduate students can claim today.

To my parents, I would like to thank you for your constant help and support as well as for you ever helpful advice given throughout all of my past, current, and future. I would not be where I am today without you both literally and figuratively.

To my grandparents and my baba, your constant support and encouragement has been a blessing to me throughout all my endeavors and studies.

To my brother Zach, your support and advice have been so helpful to me over the years, and you have always been there for me.

To my good friend Travis, you have been enormous amount of help over the years whether it be providing help on my research projects, providing an ear to hear out some of my problems, or just providing yourself so I have a lab buddy in order to perform certain lab experiments such when I had to run all those Langmuir-Blodgett trough 
samples for a research project for Dr. Hagen. You have been and always shall be my friend.

I would like to acknowledge the Bill Moore Coatings Fellowship as well as the Arther C. Edwards Endowment for the support of my stipend and the Baker and Koob Endowments for Student Success and Project-Based Learning for support of my research. In addition, I would also like to acknowledge the Western Coatings Technology Center for providing the facilities wherein I could learn and research polymer chemistry.

To Dr. Fernando, thank you for both accepting me into the Polymers and Coating Science and for your support throughout my education. Your help and support has been incredibly helpful, and, through your classes, I have gained a stronger appreciation for the science behind both polymers and coatings.

To Dr. Martinez, thank you for all your help and support over the years. Your advice has been so helpful over the quarters I have been at Cal Poly, and, through your help, I have been able to have some great experiences, such as the trip to the University of Florida, which I will always remember. You are an awesome professor, an awesome mentor, and one of the reasons why Cal Poly and the Department in particular is such an amazing institution of learning. 


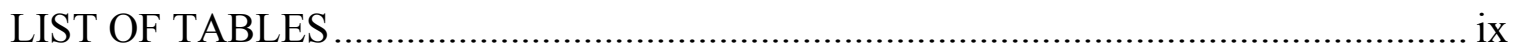

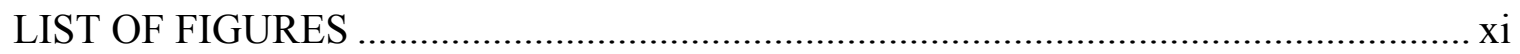

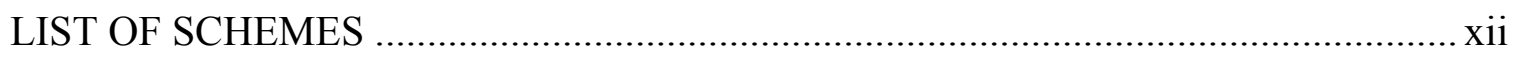

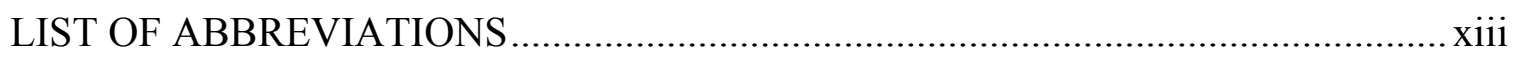

CHAPTER

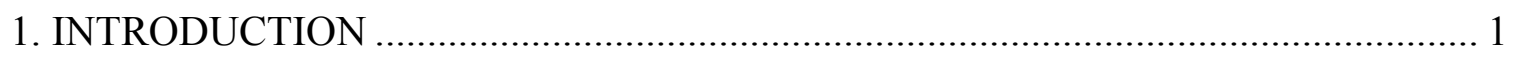

1.1 Paper Based Microfluidic Devices ...................................................................... 3

1.1.1 Device Types .......................................................................................... 5

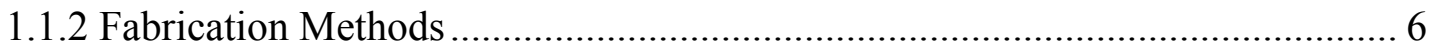

1.1.3 Chronometric Assays................................................................................... 7

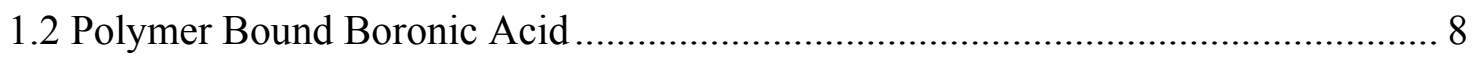

1.3 Chronometric Polymer Assay ……….............................................................. 12

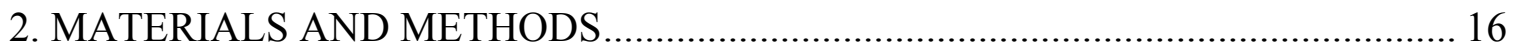

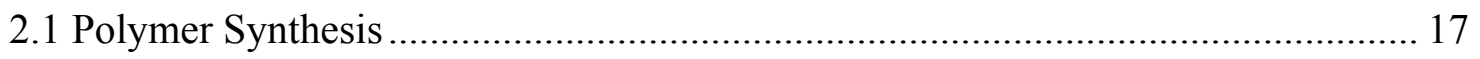

2.2 Polymer Solution Preparation ............................................................................. 18

2.2.1 Initial Aqueous Solutions ............................................................................... 19

2.2.2 Methanol Solutions ................................................................................... 19

2.2.3 Final Aqueous Solutions........................................................................ 20

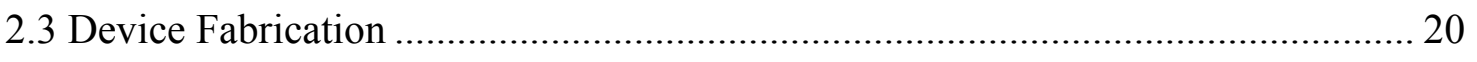

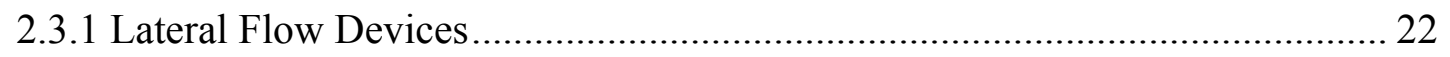

2.3.2 Vertical Flow Devices ……………………............................................ 23

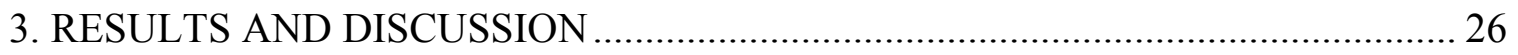




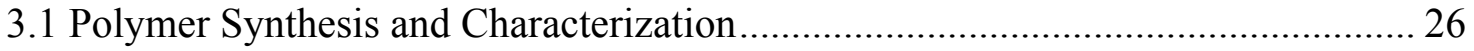

3.2 Polymer Solution Preparation and Characterization ........................................... 26

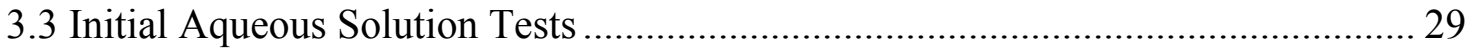

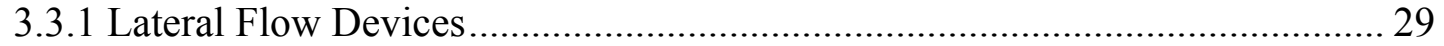

3.3.2 Vertical Flow Devices ......................................................................... 37

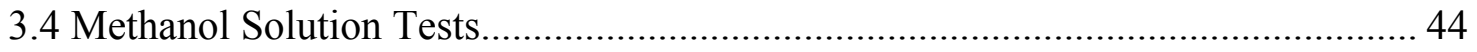

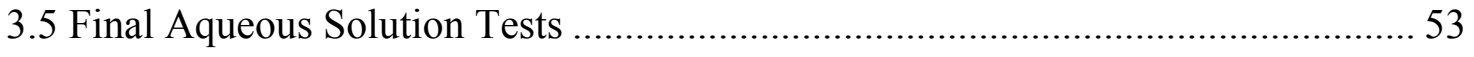

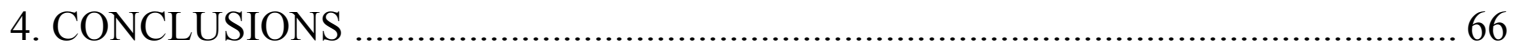

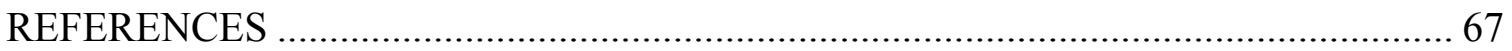




\section{LIST OF TABLES}

Table Page

Table 1: Prepared Polymer Stock Solutions and Dilutions........................................... 18

Table 2: Polymer Molecular Weight Distribution for the Initial PAPBA sample............ 26

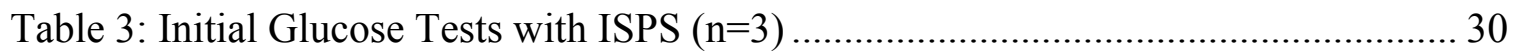

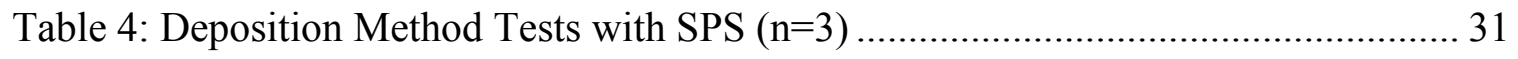

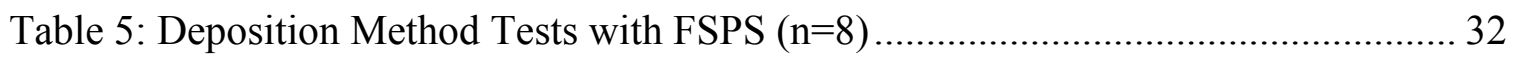

Table 6: Glucose Tests with FSPS using the Plain Polymer Deposition $(n=3)$............... 32

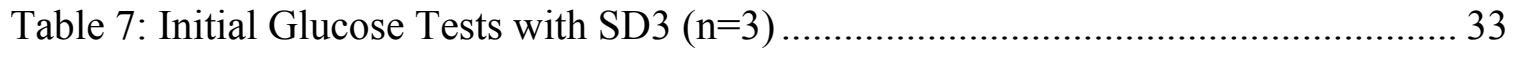

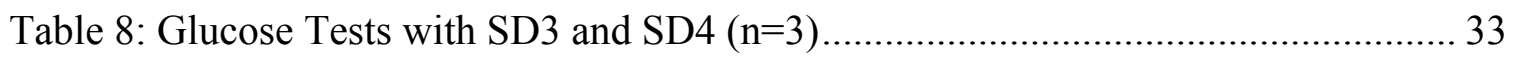

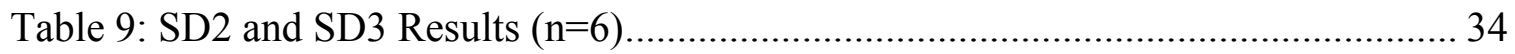

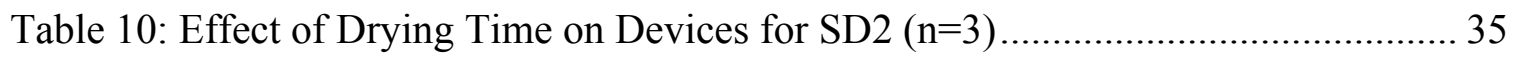

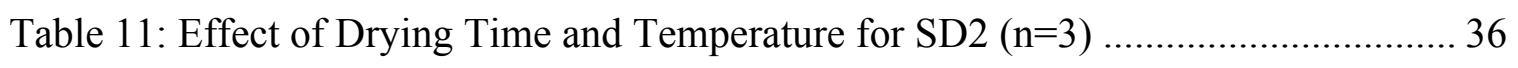

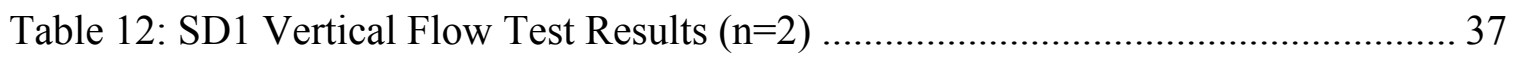

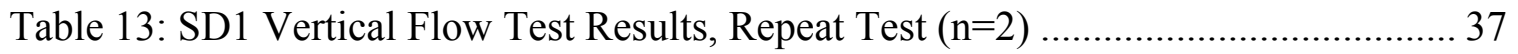

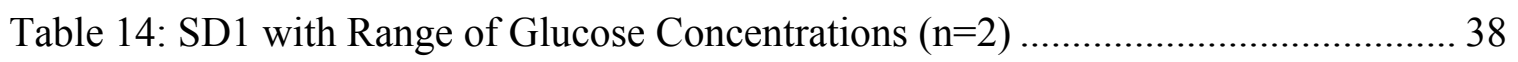

Table 15: Comparison of Normal Polymer Wells with Enlarged Polymer Wells ( $n=2)$.. 39

Table 16: SD3 Test with Glucose Concentration Range $(n=2)$.................................... 40

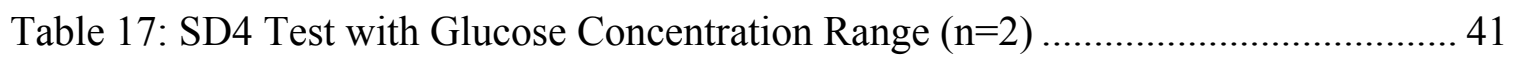

Table 18: NanoPure water, Acid, Base, and Glucose Test Results $(n=4)$....................... 41

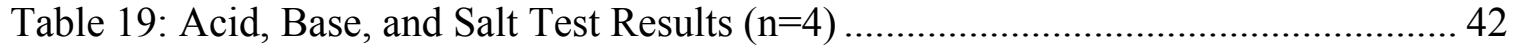

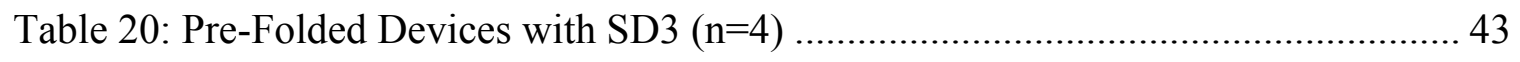

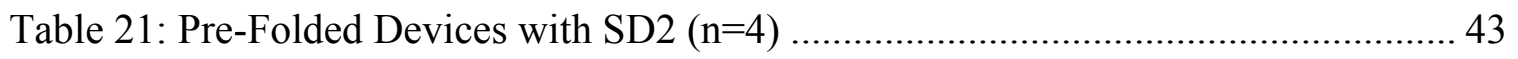

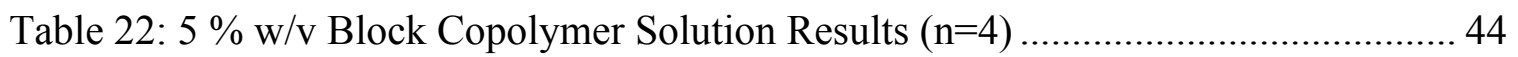

Table 23: 3.88 \% w/w Methanol Solution Devices $(\mathrm{n}=4)$........................................... 45

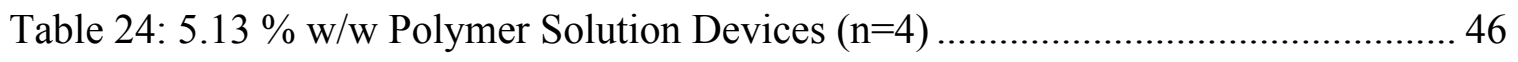

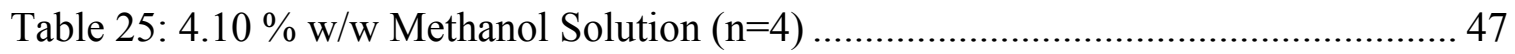

Table 26: Lateral Flow Test for 3.40 \% w/w Polymer Solution $(n=3)$........................... 48

Table 27: Lateral $(n=3)$ and Vertical $(n=4)$ Flow Tests for $1.61 \%$ w/w Solution ........... 50

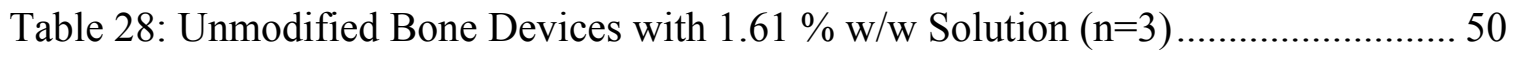

Table 29: Effect of Drying Temperature on PBBA Reactivity, Test $1(\mathrm{n}=4) \ldots \ldots \ldots \ldots \ldots \ldots . . . . . . .52$ 
Table 30: Effect of Drying Temperature on PBBA Reactivity, Test $2(\mathrm{n}=4) \ldots \ldots \ldots \ldots \ldots . . . . . . . .52$

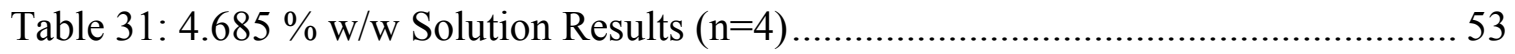

Table 32: Soak Method Results for 4.685 \% w/w Polymer Solution $(n=4)$.................... 54

Table 33: Wash Through Results for 0.595 \% w/w Polymer Solution $(n=4) \ldots \ldots \ldots \ldots \ldots . . . . .56$

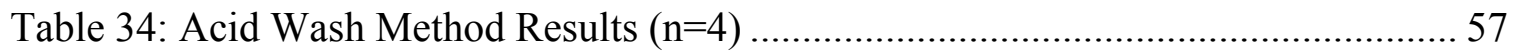

Table 35: Wash Through Testing with Bone Devices $(n=3)$...................................... 58

Table 36: Nitrocellulose and Printer Paper Tests

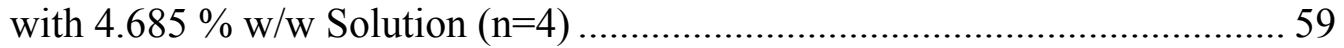

Table 37: Nitrocellulose and Printer Paper Tests with $2 \mu \mathrm{L}$ of Solution $(n=4) \ldots \ldots \ldots \ldots . . . .59$

Table 38: Nitrocellulose and Printer Paper Test Results

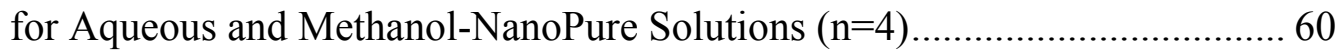

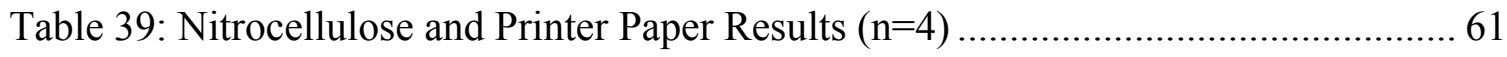

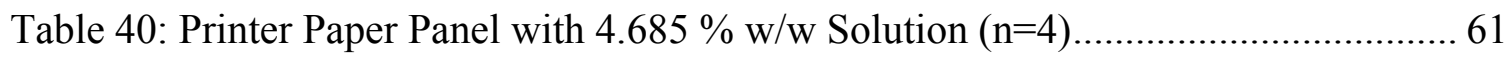

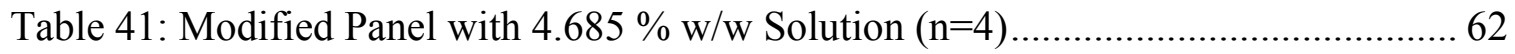




\section{LIST OF FIGURES}

Figure

Page

Figure 1: Examples of Paper-Based Microfluidic Devices ${ }^{3}$......................................... 4

Figure 2: Traditional Bone Devices ............................................................................. 5

Figure 3: Fabrication Methods for Paper Based Microfluidic Devices. ${ }^{3}$............................ 6

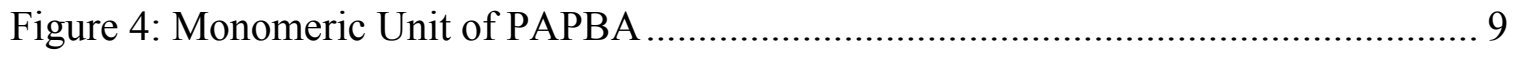

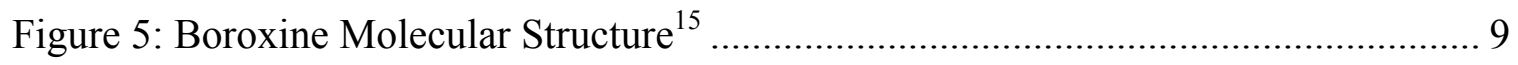

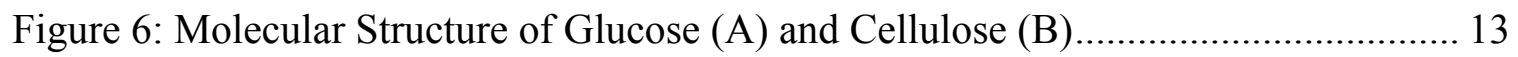

Figure 7: Molecular Structure of Nitrocellulose .......................................................... 14

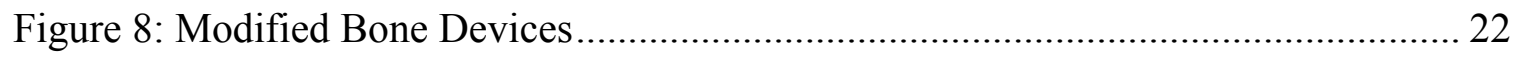

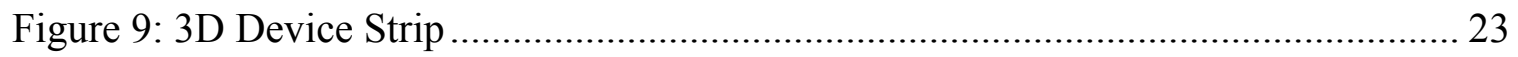

Figure 10: Assembled 3D Devices with no Polymer Deposited..................................... 23

Figure 11: Example 3D Device with Sample Flow traced through Device..................... 24

Figure 12: DLS Results for Four SD Solutions ....................................................... 28 


\section{LIST OF SCHEMES}

$\begin{array}{lll}\text { Scheme } & \text { Page }\end{array}$

Scheme 1: Reaction of Boronic Acid in Aqueous Solution ${ }^{1}$......................................... 10

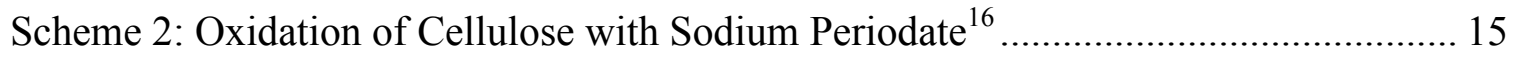




\section{LIST OF ABBREVIATIONS}

${ }^{1}$ H NMR: Proton Nuclear Magnetic Resonance

2-APBAE: 2-acrylamidophenylboronic acid pinacol ester

2D: Two-Dimensional

3-APBA: 3-acrylamidophenylboronic acid

3D: Three-Dimensional

ACPBAE: (4-((2-acrylamidoethyl)carbamoyl)phenyl)boronic acid ester

AIBN: Azobisisobutyronitrile

Avg.: Average

CRP: Controlled Radical Polymerization

DMAC: Dimethylacetamide

FSPS: Final Stock Polymer Solution

GOX: Glucose Oxidase

GPC: Gel Permeation Chromatography

HRP: Horseradish Peroxidase

ISPS: Initial Stock Polymer Solution

MicroPAD: Microfluidic Paper-Based Analytical Device

PAPBA: Poly(3-acrylamidophenylboronic acid)

PBBA: Polymer Bound Boronic Acid

PBS (1XPBS and 10XPBS): Phosphate-Buffered Saline

PDI: Polydispersity Index 
POC: Point-of-Care

RAFT: Reversible Addition-Fragmentation Chain Transfer

RLS: Resource Limited Setting

SD1: Serial Dilution 1

SD2: Serial Dilution 2

SD3: Serial Dilution 3

SD4: Serial Dilution 4

SD5: Serial Dilution 5

SPS: Stock Polymer Solution

StDev.: Standard Deviation 


\section{INTRODUCTION}

The past decades have produced great strides within the fields of medicine and biomedical engineering. New and improved diagnostic tools allow for both easier detection and identification of disease, while new treatment techniques allow doctors to combat diseases more effectively than ever before. However, despite all of the advances in health care, people in resource limited settings (RLS), such as rural and remote areas in developed or developing countries around the world, typically do not have access to the point-of-care (POC) diagnostic technologies that doctors in urban hospitals take for granted. The reason is that many of the current medical diagnostic techniques are dependent on an established medical infrastructure stocked with expensive medical equipment and operated by trained personnel, all of which are lacking in an RLS environment. So, doctors in RLS environments have to rely on symptomatic diagnosis or simple qualitative diagnostic tests.

Diagnosis of disease based on exhibited symptoms or simple tests often results in misdiagnosis, which leads to either improper treatment or a diagnosis reached too late into the life cycle of the disease-many diseases will not start to display symptoms until the disease has reached an advanced stage of incubation within the patient. If the patient is misdiagnosed, any medication that is administered to combat the disease will be wasted and might result in adverse health effects for the patient. On the other hand, if the patient is diagnosed at too late a stage in the lifecycle of the disease, the treatment may not be as effective or may result permanent health effects for the patient. Thus, one way to increase both the effectiveness and the quality of medical treatment for patients in an RLS 
environment is through the development of cheap, efficient, and easy-to-use diagnostic tools that will provide quantitative results.

A relatively new platform for the development of simple diagnostic tools is paper based microfluidic devices or microPADs (Figure 1). MicroPADs are devices made out of paper or other porous membranes that are patterned with hydrophobic inks to produce networks of hydrophilic channels and test zones that wick fluids by capillary action and can be used to conduct assays. MicroPADs are cheap to produce, easy to use, and highly portable due to their small size and mass. These devices can perform multiple assays simultaneous with only a minimal amount of sample, such as blood or urine. Finally, disposal of these devices is a straightforward process as they can be incinerated because they are made primarily out of paper.

However, microPADs often rely on enzymatic reactions in order to produce their results. For example, assays for glucose on microPADs usually involve the coupled enzymatic reactions of Glucose Oxidase (GOX) and Horseradish Peroxidase (HRP) in order to produce a colorimetric signal. The problem with using enzymes in POC devices is that they require carefully controlled storage conditions in order to maintain their activity, ${ }^{1}$ otherwise the device will not provide consistent results. To overcome this limitation, one could either devise better ways to stabilize the enzymes in the devices or determine a viable non-enzymatic assay that could serve as reliable substitute, while still providing the required quantitative results necessary for POC diagnosis. Focusing on the later solution, the main objective of this research project was to investigate the use of polymers as an alternative to enzymes for detecting analytes using microPADs. 
As a first step, polymer-bound boronic acid (PBBA), a glucose and $\mathrm{pH}$ responsive polymer, was investigated for developing a chronometric paper-based glucose assay. PBBA is insoluble when the $\mathrm{pH}$ is below $\sim 9$ (the $\mathrm{pK}_{\mathrm{a}}$ of boronic acid), but becomes soluble in water when the $\mathrm{pH}$ is raised above 9 or in the presence of glucose. ${ }^{1,2}$ By depositing PBBA in the middle of a paper-based channel, it was hypothesized that water wicking across the channel would stop or at least slow down, when it encountered the polymer, but that a solution of glucose would dissolve the polymer and would wick across the channel at a rate that was proportional to the concentration of glucose in the sample - that is, solutions containing higher concentrations of glucose would take less time to wick across the channel. Therefore, the specific objective of this research project was to develop a glucose assay using PBBA analyzed by chronometric measurements that is, the signal for the assay would be the amount of time required for a sample to wick across a channel in a microPAD.

\subsection{Paper Based Microfluidic Devices}

Paper-based chemical testing, in its most basic form, has been around for centuries, with the first example being the use of litmus paper to semi-quantitatively determine the $\mathrm{pH}$ of an aqueous solution using a colorimetric signal. In fact, the initial paper-based testing devices were simply strips of paper that had been treated with reagents to produce a color change in the presence of an analyte. ${ }^{3}$ The first paper-based diabetes dipstick test was proposed during the 1950's and introduced commercially in the 1960's. These first microPADs were only able to perform one test at a time and were only able to produce either a qualitative result, such as whether the patient was pregnant or not, or a semi-quantitative result, such as a blood glucose concentration. For semi- 
quantitative results, the color produced by the assay would be compared to a color chart supplied with the device. ${ }^{4}$

Paper-based microfluidic devices, or microPADs, (Figure 1) are the newest generation of paper-based diagnostic devices that incorporate hydrophilic channels and reaction zones bounded by hydrophobic barriers that are patterned into a piece of paper. Simple methods for patterning paper have enabled the development of more complex devices with intricate networks of channels and test zones that can perform multiple sample processing steps.

A

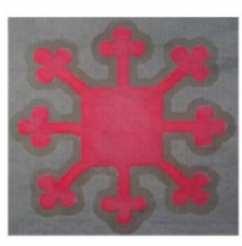

$\mathbf{F}$

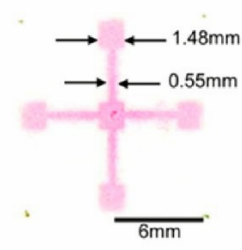

$\mathbf{J}$

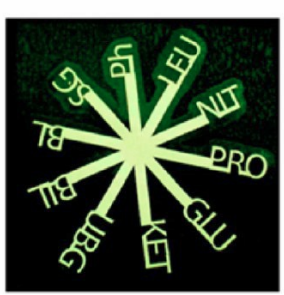

B

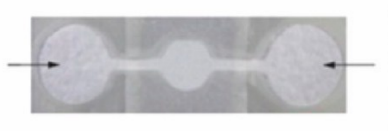

G

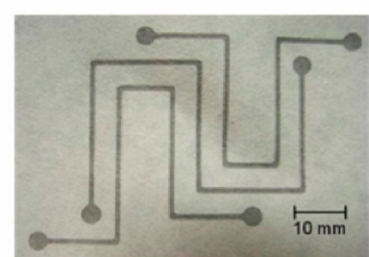

$\mathbf{K}$

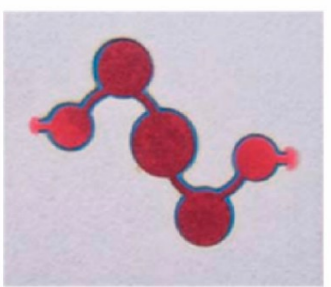

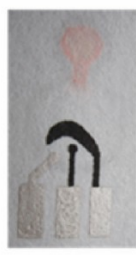

H

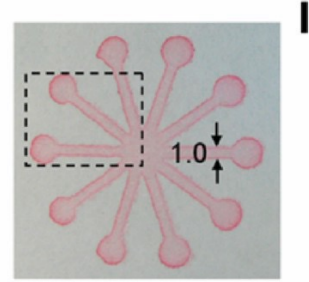

$\mathbf{L}$
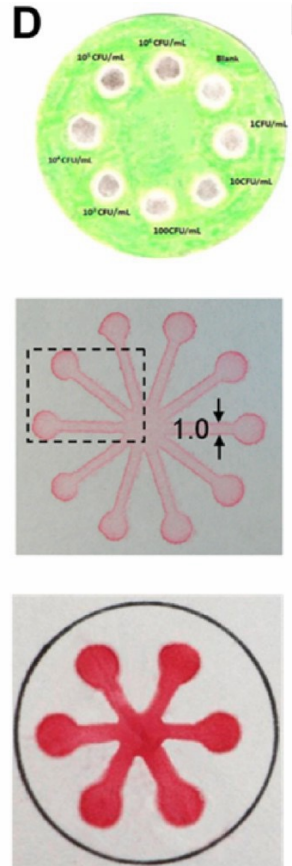

I
E
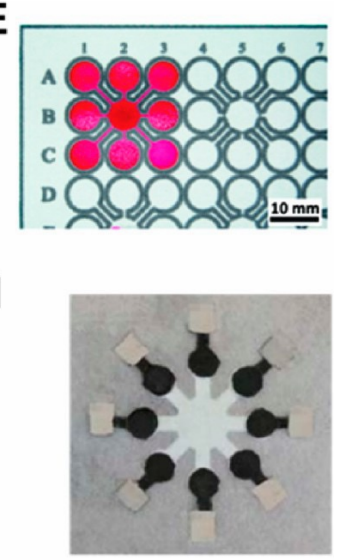

M

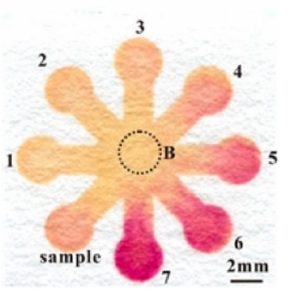

Figure 1: Examples of Paper-Based Microfluidic Devices ${ }^{3}$ Fabricated by:

A - Wax Stamping with movable type printing. B - Wax Dipping. C - Screen-Printed Wax Device and Electrodes. D - Wax Drawing through a stencil. E - Wax Printing. F - Inkjet Etching of Polystyrene in Paper with Toluene. G - Inkjet Printing of Alkyl Ketene Dimer (AKD). H - Flexographic Printing of Polystyrene. I - Photoresist Patterning with Screen-Printed Electrodes. J - Computer Controlled Knife Cutting in Nitrocellulose. K - Laser-Cut Hollow Channels. L - Vapor-Phase Polymer Deposition. $\mathrm{M}$ - Chemical Modification with Alkylsilane self-assembling and $\mathrm{UV} / \mathrm{O}_{3}$ patterning. Reprinted with permission from ref 3. Copyright 2014 American Chemical Society. 


\subsubsection{Device Types}

Two basic types of microPADs are currently being utilized, two-dimensional (2D) lateral-flow devices and three-dimensional (3D) vertical flow devices. 2D devices are made from a single layer of paper, while 3D devices are made by stacking multiple 2D devices on top of each other. The layers of paper in the 3D device can either be bonded to each other permanently using permanent adhesives, or they can be held together temporarily using a manifold or removable adhesives. Of these two types of devices, 2D devices are, so far, more common because they are relatively easy to make using a broad array of fabrication techniques and still allow for a fairly broad range of customization through modifications to the device's pattern, the barrier material, and the reagent deposition method.

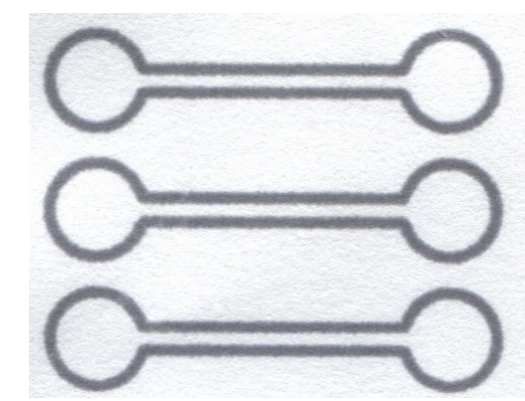

Figure 2: Traditional Bone Devices

The most common 2D device is the "bone" device which is composed of two circular hydrophilic zones connected by a hydrophilic channel (Figure 2). One end of the device serves as the inlet zone where the sample is introduced to the device, and the other end of the device serves as the results zone, where the colorimetric signal from the assay can be recorded. Many of the current 2D device patterns being developed and produced are modifications of this basic design. 3D devices, on the other hand, allow for a much 
higher channel density to be incorporated into the device due to the multi layer structure, so more complex sample processing is possible in this format.

\subsubsection{Fabrication Methods}

MicroPADs are currently produced using a variety of methods that can be grouped into the four fabrication methods depicted in Figure 3: handcrafted, printed, formed using masks, or formed through cutting and shaping the substrate. While each fabrication method has its own advantages and disadvantages, they all ultimately lead to the production of hydrophilic channels and test zones in paper that can wick fluids by capillary action.

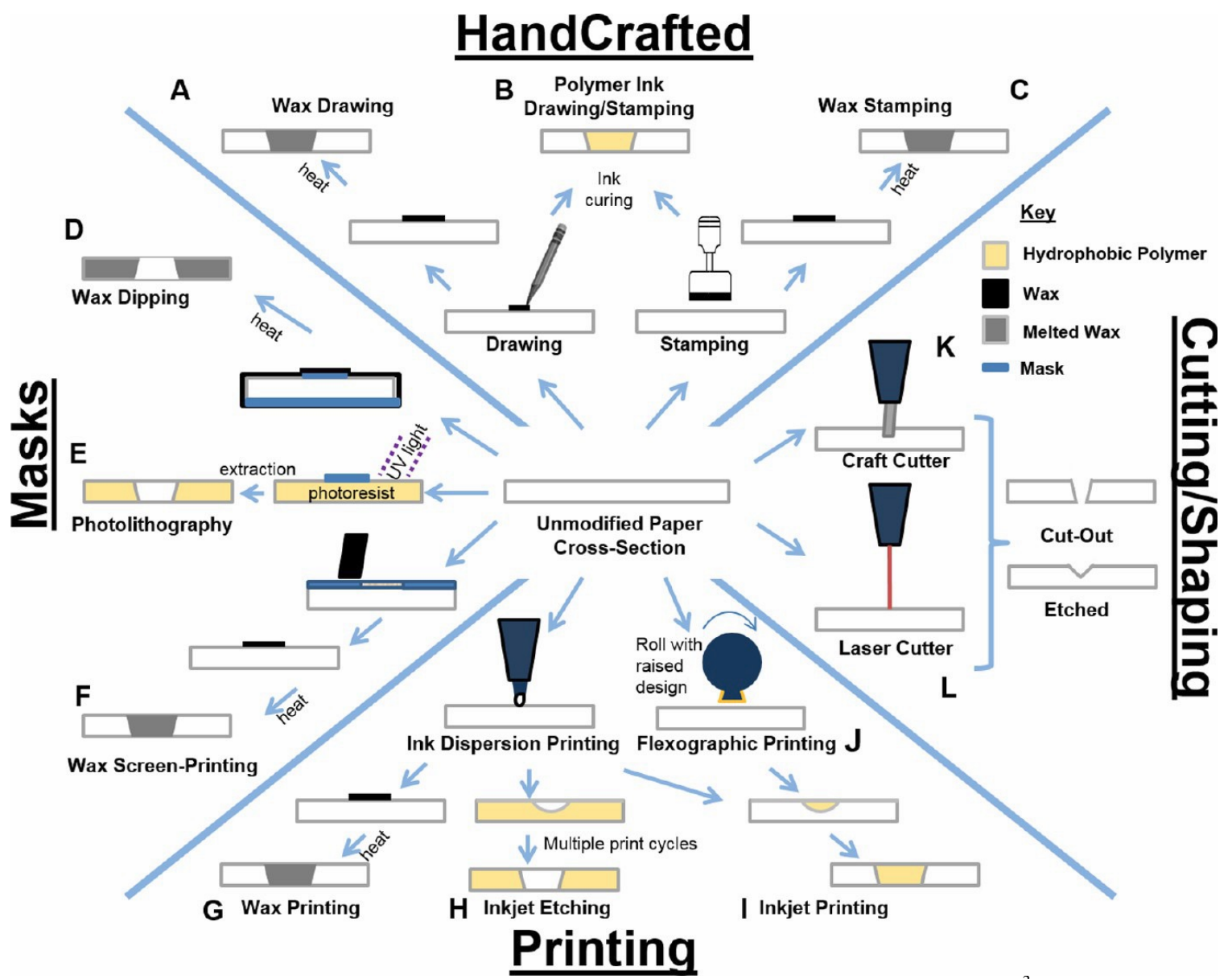

Figure 3: Fabrication Methods for Paper Based Microfluidic Devices. ${ }^{3}$

Reprinted with permission from ref 3. Copyright 2014 American Chemical Society. 
For this research project, the method of Wax Printing was selected for fabricating microPADs. Wax printing is an additive fabrication method where, as the name suggests, wax is printed on the surface of a piece of paper and then melted so that it penetrates through the thickness of the paper to create hydrophobic barriers (Figure 3G). This technique is currently the most common fabrication method for microPADs and uses a commercially available office printer that prints a wax-based ink (Figure 1E). ${ }^{5,6}$ This method offers a wide range of customizability as new device designs can be created on a computer and then fabricated within minutes.

\subsubsection{Chronometric Assays}

One important consideration when developing a diagnostic assay is the type of signal that is generated by the assay. Colorimetric assays are a common choice for POC devices because the results can be visualized without the aid of any supporting equipment or instrumentation. However, colorimetric assays are typically not very sensitive and generally only work well for a limited range of concentrations of analyte. Other detection techniques that have been demonstrated on microPADs include electrochemistry, absorption, fluorescence and chemiluminescence. While these techniques tend to be more sensitive than colorimetric assays, they require supporting instrumentation in order to obtain the signal. A less conventional detection technique that has high sensitivity and only requires minimal instrumentation, namely a timer, is chronometric detection. The signal for chronometric paper-based assays is the time that it takes for the sample to wick from one designated location on the device to another. By incorporating specific reagents into the device that react with the analyte, it can be possible to influence the wicking rate 
through the device so that the signal (i.e., time) is a function of the concentration of analyte in the sample.

Chronometric paper-based assays were pioneered by the Phillips group at Pennsylvania State University. For their assays, Phillips et al. developed a series of hydrophobic self-immolative polymers that, upon reacting with hydrogen peroxide, would depolymerize into water-soluble monomers. They then applied these polymers to one layer of a 3D microPAD and demonstrated that the time it would take a sample to wick through the device was inversely proportional to the concentration of hydrogen peroxide in the sample. Then, by using the reaction of glucose and glucose oxidase to produce hydrogen peroxide, the Phillips group demonstrated the detection of glucose oxidase at femtomolar concentrations and showed that their assay was not affected by variations in sample volume, sample viscosity, or variations in environmental conditions such as temperature and humidity. ${ }^{7}$

\subsection{Polymer Bound Boronic Acid}

The chronometric paper-based assays demonstrated by the Phillips group were sensitive and robust, but still relied on enzymatic reactions. By developing POC diagnostic assays that rely exclusively on polymers for detection, it may be possible to extend the shelf life and reduce the cost of the devices. In addition, because polymers are synthetic reagents, the polymer specifications could easily be tuned to meet changing device and assay requirements. Devices relying on polymers for detection could, in theory, be hardy enough to survive both long storage periods and extreme changes in environment (temperature and humidity) while still producing the quantitative results that are needed for successful diagnosis. The research presented in this thesis focuses on the 
use of Polymer Bound Boronic Acid (PBBA) for detection of glucose on microPADs (Figure 4).

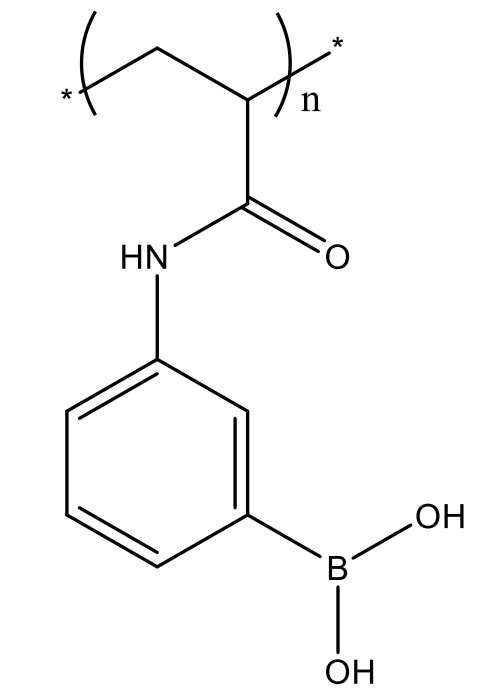

Figure 4:Monomeric Unit of PAPBA

Chemical structure of Poly(3-acrylamidophenylboronic acid) (PAPBA), the specific PBBA used for this research project.

Boronic acid functional groups react with diols, and with glucose in particular, with a high affinity. By covalently bonding boronic acid to a polymer backbone, it is possible to create glucose responsive polymers that are soluble in the presence of glucose and are insoluble in the absence of glucose. Under acidic or neutral conditions, the PBBA chains can chemically crosslink via boroxine linkages, depicted above in Figure 5, due to the reactivity of the boronic acid functional groups.

\section{Boroxine}

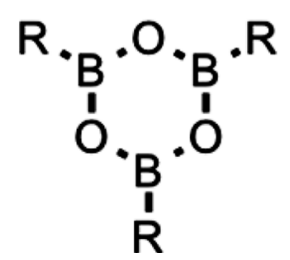

Figure 5: Boroxine Molecular Structure ${ }^{15}$ 
This forms a continuous hydrophobic network that is insoluble in water. Under basic conditions though, the boron can act as an electrophile and react with hydroxide in the solution leading to a water-soluble anionic product as denoted in Scheme 1. The addition of a diol, especially such as one of the possible diols present on glucose, to PBBA will shift the equilibrium of the reaction so that the anionic product is favored. This shift breaks up the crosslinked PBBA and causes the polymer to undergo a hydrophobic to hydrophilic transition in water.

For a simple boronic acid compound, this shift will happen rapidly with measured rate constants for phenylboronic acid determined to be at approximately $10^{2}-10^{3} \mathrm{M}^{-1} \mathrm{~s}^{-1}$. However, this rate constant is only for a simple boronic acid and, as such, is only an approximation. The Sumerlin group has done solution studies with a PBBA block copolymer using dynamic light scattering (DLS) to determine the dissociation time for various glucose concentrations. The PBBA demonstrated a slower reaction time as the polymer reacted with the glucose in a matter of 1-2 hours depending on the glucose concentration of the solution. ${ }^{1}$ However, it should be noted that these tests were performed using a block copolymer in solution and were analyzed using DLS, and thus, the results can only serve as a rough approximation for the reaction of the PBBA within the context of the microPAD.

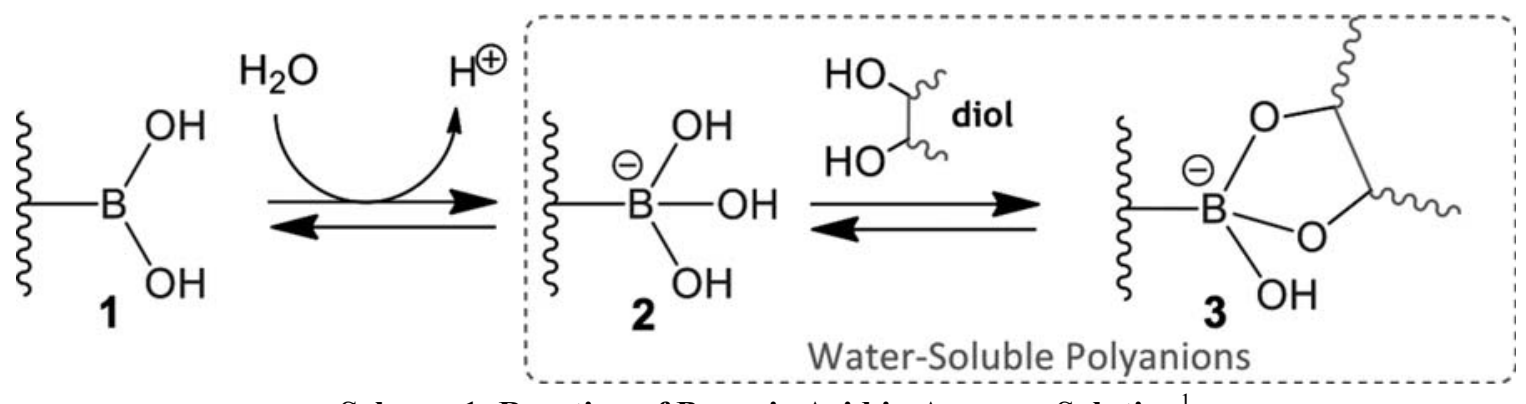

Scheme 1: Reaction of Boronic Acid in Aqueous Solution ${ }^{1}$ 
The polymer chain backbone yields several advantages over a pure boronic acid compound for analytical measurement. The polymer chain helps to stabilize the boronic acid and allows the boronic acid functional groups to react with each other to form a solid barrier under certain conditions. In addition, the polymer chain also allows the analyte responsiveness of the boronic acid functional group to be tuned through modifications to the molecular weight, the monomeric composition, or the molecular weight distribution by simply changing parameters for the synthesis of the polymer. Finally, the polymer backbone and the boronic acid side chains can also be modified to produce polymers with a wide range of properties.

The glucose responsiveness of PBBA has been investigated in solution by the Sumerlin group at the University of Florida. ${ }^{1,2}$ Their studies focused on characterizing the aggregation and dissociation of PBBA with changes in $\mathrm{pH}$ and concentration of glucose. The solution properties were modified in order to design polymer aggregates that were capable of self-assembly in response to changes in the solution. These aggregates were used to both solubilize as well as deliver model hydrophobic compounds through a controlled release mechanism. The resulting aggregates were detected using UV-Vis spectroscopy and fluorescence. The PBBA dissociation was induced by either raising the $\mathrm{pH}$ of the solution above the $\mathrm{pK}_{\mathrm{a}}$ of the boronic acid residues or by adding sugars to the solution. The work presented in this thesis is the first example of incorporating PBBA into a microPAD for detection of glucose.

The PBBA used for this research was synthesized using a controlled radical polymerization (CRP) technique known as reversible addition-fragmentation chain transfer (RAFT) polymerization to produce a polymer with a low polydispersity index 
(PDI) and target molecular weight. ${ }^{9}$ The PDI and molecular weight of the PBBA are important for this project because they will theoretically influence the solubility of the polymer. For example, if the polymer backbone has a low molecular weight and a low PDI, the PBBA should be more soluble and may not form an effective barrier to slow wicking in the microPAD. However, a high molecular weight polymer backbone could result in a PBBA that is insoluble regardless of the concentration of glucose in the sample. By controlling the synthesis of the polymer, it should be possible to produce PBBA with a range of molecular weights and PDI's, which could be used to tune the sensitivity of the paper-based assay. The target was to develop a device that could perform a glucose assay within the span of about 30 minutes and would be sensitive enough to detect minor differences of $\sim 1 \mathrm{mM}$ for glucose concentrations between 0.5 $\mathrm{mM}$ and $20 \mathrm{mM}$.

\subsection{Chronometric Polymer Assay}

By combining the PBBA assay with a chronometric analysis method, the hypothesis for this project was that the polymer would serve as a temporary barrier within the microPAD for the sample and would gradually dissolve over a period of time proportional to the concentration of glucose in the sample - that is, solutions containing higher concentrations of glucose would take less time to dissolve the barrier and therefore to finish. However, the deposition of the polymer altered several of the properties of the paper substrate. The contact angle, surface energy, and capillary flow of water on and within the paper substrate were all changed through the addition of the polymeric material within the cellulosic matrix. 
The best way to quantify the shift in the capillary flow through the addition of the polymer would be to test the polymer powder using the capillary rise method. For this procedure, the powder is packed into a capillary tube that is $1 \mathrm{~cm}$ in diameter and treated as a bundle of thin capillaries, and a reference fluid that is completely wetting is run through this tube while either the speed of the fluid rise or the pressure necessary to keep the fluid out of the powder is measured. Either measurement technique will allow the user to effectively calculate the contact angle for the polymer. However, one limitation to this method is that it takes the average over many particles. In addition, the size distribution remains unknown as it cannot be calculated using this method. Finally, this method relies on the assumption that a powder can be simply treated as a bundle of capillaries and is also dependent on the specific theoretical model that is applied to the system. ${ }^{10}$

One other potential problem that was foreseen with this research project was interference from the cellulosic material. Both the glucose (Figure 6A) and the cellulose (Figure 6B) have diol sites that could potentially react with the PBBA.

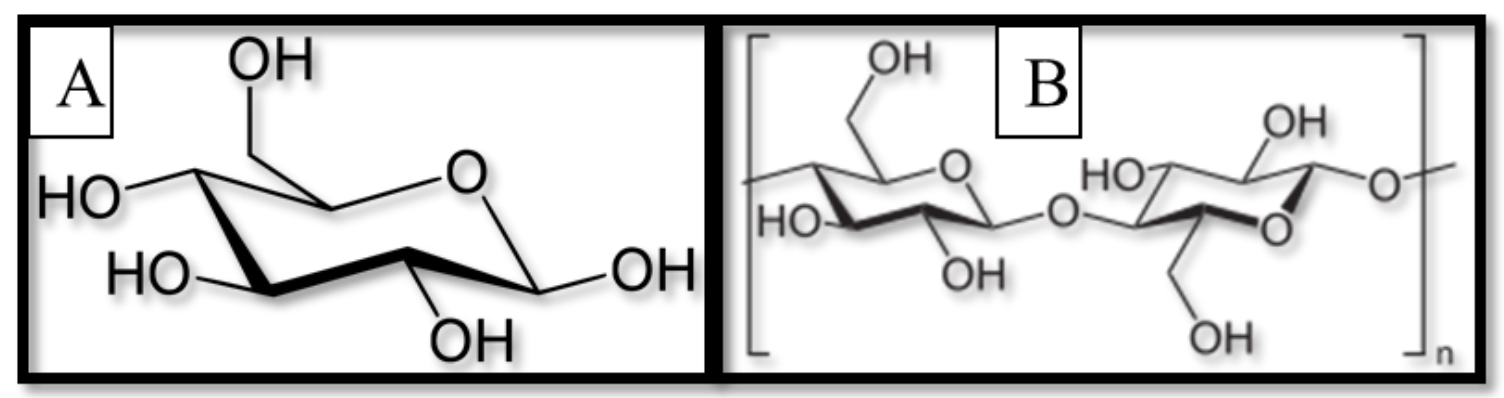

Figure 6: Molecular Structure of Glucose (A) and Cellulose (B) 
The potential reaction between the cellulose of the substrate and the PBBA could interfere with the reaction between the glucose sample and the PBBA reducing the overall effectiveness of the glucose assay. As a result, select panels of the 3D devices were either fabricated using different substrate materials such as nitrocellulose (Figure 7) and printer paper panels, or were modified by using sodium periodate to oxidize the alcohol groups of the diol on the cellulose to aldehyde groups.

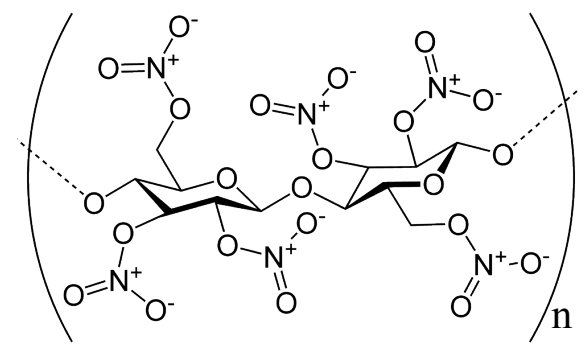

Figure 7: Molecular Structure of Nitrocellulose

Nitrocellulose, also known as gun cotton, is a modified version of cellulose wherein all the alcohol groups have been replaced with nitro groups. Nitrocellulose panels are more brittle, not quite as sturdy, and harder to fabricate than chromatography paper devices which thus made these panels more difficult to work with. However, the nitrocellulose panels no longer possess the diol that the cellulose had and therefore should not have any interfering reactions that could compromise the glucose assay.

In addition to nitrocellulose panels, printer paper panels were also investigated to possibly eliminate any side reactions between the cellulose and the glucose sample. Printer paper is fabricated with several different binders materials so that the paper is able to both remain flexible as well as maintain structural and mechanical stability. The 
hypothesis was that these binders might either interfere with the reaction between the PBBA and the cellulose or stabilize the PBBA on the substrate.

Finally, select panels were also modified by reacting them with a solution of sodium periodate in order to oxidize the diol on the cellulose. The periodate ion in particularly served as an oxidizing agent for the hydroxyl functional groups oxidizing them to aldehyde functional groups as denoted in Scheme 2. However, this particular reaction needs to be isolated from sunlight as the UV light can terminate the reaction. ${ }^{11}$

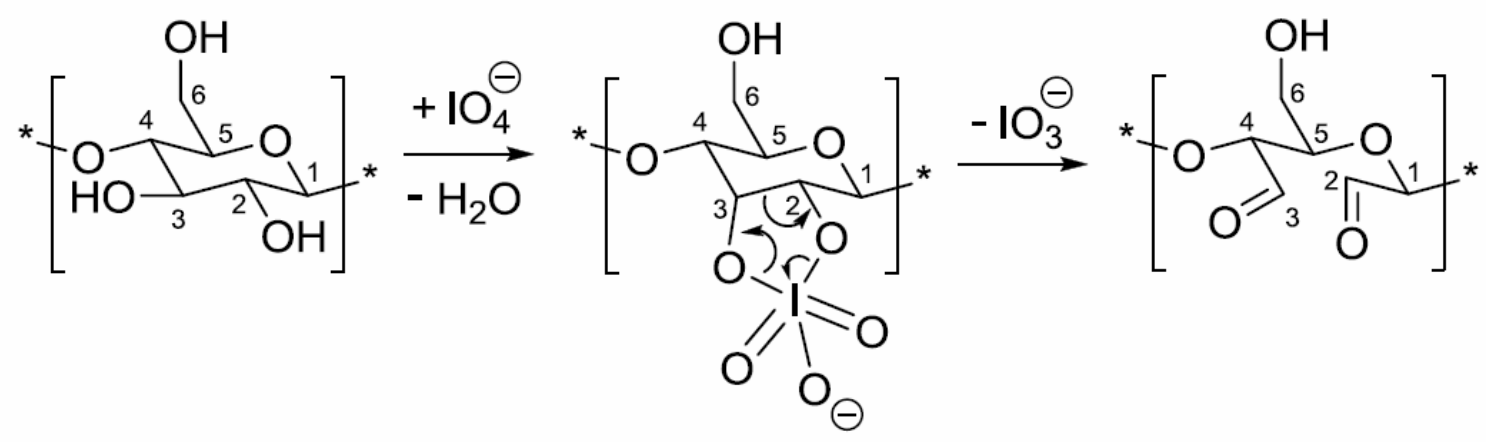

Scheme 2: Oxidation of Cellulose with Sodium Periodate ${ }^{16}$ 


\section{MATERIALS AND METHODS}

All of the reagents used for this research were purchased from commercial sources unless stated otherwise. The following chemicals were used: 1N Hydrochloric Acid (Fisher Scientific), 1N Sodium Hydroxide (Fisher Scientific), Color Dyes (Red, Green, and Blue dyes obtained from General Chemistry Stockroom), PAPBA (synthesized by the Sumerlin Group at the University of Florida), Solid Sodium Hydroxide (Polymers Laboratory), Certified ACS Pure (12.1 M) Hydrochloric Acid (Fisher Scientific), Glucose (Sigma Aldrich), Sodium Periodate (J.T. Baker Chemical Company, Alfa Aesar), Potassium Periodate (J.T. Baker Chemical Company), Glacial Acetic Acid (General Chemistry Stockroom), Sodium Chloride (General Chemistry Stockroom), and HPLC Grade Methanol (Fisher Scientific). A 1X phosphate-buffered saline solution (1XPBS) was prepared from a stock 10XPBS solution (Fisher Scientific) using in-house NanoPure water obtained from a NanoPure Dispenser (Thermo Scientific Barnstead NanoPure Filtration System). All devices were printed on either chromatography paper (Whatman No. 1), a nitrocellulose membrane (Whatman Protran BA 85), or commercially available printer paper.

Two glucose stock solutions were prepared. One solution used NanoPure water as the solvent, and the other solution used 1XPBS as the solvent. Seven serial dilutions were made from each stock solution and were stored in microcentrifuge tubes at $4{ }^{\circ} \mathrm{C}$ until they were used. The following concentrations of the 1XPBS-Glucose solutions were prepared: $108.1 \mathrm{mM}, 54.05 \mathrm{mM}, 27.1 \mathrm{mM}, 13.53 \mathrm{mM}, 6.763 \mathrm{mM}, 3.381 \mathrm{mM}, 1.691 \mathrm{mM}$, and $0.845 \mathrm{mM}$. The following concentrations of the NanoPure-Glucose solutions were 
prepared: $101 \mathrm{mM}, 50.5 \mathrm{mM}, 25.3 \mathrm{mM}, 12.65 \mathrm{mM}, 6.325 \mathrm{mM}, 3.163 \mathrm{mM}, 1.58 \mathrm{mM}$, and $0.791 \mathrm{mM}$. These glucose solutions were used throughout the course of the project.

\subsection{Polymer Synthesis}

The PAPBA (Figure 4) was provided for this research project by the Sumerlin research group at the University of Florida. The polymer was synthesized via RAFT polymerization and was analyzed using Gel Permeation Chromatography by the Sumerlin group. In addition, the polymer was purified via dialysis and lyophilized before use by the Sumerlin group as well. Gel Permeation Chromatography (GPC) ${ }^{12}$ ia a size exclusion chromatography method that is used to determine the molecular weights and the PDI of a polymer sample.

Four additional PBBA samples were produced in collaboration with the Sumerlin group using the following monomers: 3-acrylamidophenylboronic acid (3-APBA), 2-acrylamidophenylboronic acid pinacol ester (2-APBAE), (4-((2-acrylamidoethyl) carbamoyl)phenyl)boronic acid ester (ACPBAE), and Urea. These polymer samples were produced using a simple free-radical polymerization method unlike the CRP method (RAFT) that was used to produce the initial sample. The primary difference between each of the polymer samples was the monomeric repeat unit. The monomers were polymerized in dimethylacetamide (DMAC) with azobisisobutyronitrile (AIBN) as the initiator and with trioxane added to monitor the reaction conversion. The reaction vials were purged with Argon gas for 30 minutes and refluxed for $\sim 18$ hours at $70^{\circ} \mathrm{C}$. The PBBA samples were both characterized using GPC and Proton Nuclear Magnetic Resonance ( ${ }^{1} \mathrm{H}$ NMR) and purified through dialysis in a $0.1 \mathrm{M} \mathrm{NaOH}$ solution for several days by the Sumerlin group. 3,500 molecular weight dialysis tubing was used for both the 2-APBAE and the 
ACPBAE polymers, and 8,000 molecular weight dialysis tubing was used for both the Urea and the 3-APBA polymers. After dialysis, the polymers were lyophilized to dry them and render each sample into a powder. Unfortunately, the samples were not processed in time to be used for testing.

\subsection{Polymer Solution Preparation}

Table 1: Prepared Polymer Stock Solutions and Dilutions

\begin{tabular}{|c|c|c|c|c|}
\hline $\begin{array}{l}\text { Solution } \\
\text { Type }\end{array}$ & $\begin{array}{l}\text { Initial Aqueous } \\
\text { Solutions }\end{array}$ & $\begin{array}{l}\text { Methanol } \\
\text { Solutions }\end{array}$ & $\begin{array}{c}\text { Methanol-NanoPure } \\
\text { Solutions }\end{array}$ & $\begin{array}{c}\text { Final Aqueous } \\
\text { Solutions }\end{array}$ \\
\hline $\begin{array}{c}\text { Stock } \\
\text { Solutions }\end{array}$ & $\begin{array}{c}\text { ISPS } \\
\text { SPS } \\
\text { FSPS } \\
\sim 12 \% \mathrm{w} / \mathrm{w}\end{array}$ & $7.8 \% \mathrm{w} / \mathrm{w}$ & $5.1 \% \mathrm{w} / \mathrm{w}$ & $4.7 \% \mathrm{w} / \mathrm{w}$ \\
\hline Dilutions & $\begin{array}{l}\text { SD1 } \\
\text { SD2 } \\
\text { SD3 } \\
\text { SD4 } \\
\text { SD5 }\end{array}$ & $3.9 \% \mathrm{w} / \mathrm{w}$ & $\begin{array}{l}4.1 \% \mathrm{w} / \mathrm{w} \\
3.4 \% \mathrm{w} / \mathrm{w} \\
1.6 \% \mathrm{w} / \mathrm{w}\end{array}$ & $\begin{array}{l}2.4 \% \mathrm{w} / \mathrm{w} \\
1.2 \% \mathrm{w} / \mathrm{w} \\
0.91 \% \mathrm{w} / \mathrm{w} \\
0.60 \% \mathrm{w} / \mathrm{w}\end{array}$ \\
\hline
\end{tabular}

Three different types of polymeric solutions were produced to test the effect of the solvent on the effectiveness of the polymer for glucose detection. The first polymer solutions formulated were prepared in a basic, aqueous environment in an attempt to reproduce the solution conditions used by the Sumerlin group. A second set of polymer solutions was produced using methanol as the solvent. The solutions were then further diluted with NanoPure water so that the solution would not wick across the wax barriers 
in the microPADs. Finally, a third set of aqueous solutions was formulated as well. All polymer solutions were prepared in $1.5 \mathrm{~mL}$ plastic microcentrifuge tubes. Stock polymer solutions were vortexed (Fisher Vortex Genie 2) during formulation to dissolve the polymer completely then were centrifuged (Fisher Scientific Marathon Micro A) to remove any undissolved polymer. All the various solution concentrations for the three different solution sets are noted in Table 1 .

\subsubsection{Initial Aqueous Solutions}

The initial aqueous solutions were produced in $0.1 \mathrm{M} \mathrm{NaOH}$ at a concentration of $\sim 12 \% \mathrm{w} / \mathrm{w}$ though, due to problems with the analytical balance used, the exact concentration was unknown. After initial testing with the three stock polymer solutions (ISPS, SPS, and FSPS), five serial dilutions (SD1, SD2, SD3, SD4, and SD5) were produced by combining equal volumes of the previous polymer solution and $0.1 \mathrm{M}$ $\mathrm{NaOH}$ in a clean microcentrifuge tube. These aqueous polymer solutions were all analyzed using Dynamic Light Scattering (DLS, Wyatt Technologies DynaPro NanoStar). The hypothesis was that the DLS results could be used to determine the polymer particle size distribution in solution. This would, in theory, allow the interactions between the paper and the polymer to be more clearly elucidated.

\subsubsection{Methanol Solutions}

A $5 \% \mathrm{w} / \mathrm{v}$ solution of a thermal responsive block copolymer was produced in methanol and tested with a range of glucose concentrations on the vertical flow devices. Results obtained from these tests prompted a shift in research as testing was performed using methanol based polymer solutions made from the initial polymer sample. 
A $7.8 \% \mathrm{w} / \mathrm{w}$ solution of PAPBA was also prepared in methanol. This solution was diluted with methanol to $3.9 \% \mathrm{w} / \mathrm{w}$ and $1.9 \% \mathrm{w} / \mathrm{w}$. To make sure that the polymer solution stayed contained within the wax barrier on the microPADs, the initial methanol solution was diluted with NanoPure water to a concentration of $5.13 \% \mathrm{w} / \mathrm{w}$. This NanoPure dilution was further diluted using an 80:20 solution by volume of methanol:water to $4.1 \% \mathrm{w} / \mathrm{w}, 3.4 \% \mathrm{w} / \mathrm{w}$, and $1.6 \% \mathrm{w} / \mathrm{w}$.

\subsubsection{Final Aqueous Solutions}

Due to concerns over the effect of the Methanol-water polymer solutions on the wax barriers, testing returned to using aqueous solutions. A new aqueous stock solution was prepared initially in a solution of $0.1 \mathrm{M} \mathrm{NaOH}$ though the $\mathrm{pH}$ of the solution had to be adjusted using $1 \mathrm{M} \mathrm{NaOH}$ with the final concentration of solution at $4.7 \% \mathrm{w} / \mathrm{w}$. This stock solution was further diluted with NanoPure water to yield solutions with concentrations of $2.4 \% \mathrm{w} / \mathrm{w}, 1.2 \% \mathrm{w} / \mathrm{w}, 0.91 \% \mathrm{w} / \mathrm{w}$, and $0.60 \% \mathrm{w} / \mathrm{w}$.

\subsection{Device Fabrication}

Two types of microfluidic devices, the 2D lateral flow and the 3D vertical flow devices, were tested with the PBBA assay to determine what effect, if any, the device design had on the analytical effectiveness of the non-enzymatic assay. All of the devices used for testing were designed on a computer. AutoCAD was used to design the lateral flow devices, and Adobe Illustrator was used to design the vertical flow devices. The device pattern was then printed onto chromatography paper using a solid-ink wax printer (Xerox Phaser 8560), and the printed sheets of paper were then baked in a convection oven (MTI Compact Forced Air Convection Oven) at $195{ }^{\circ} \mathrm{C}$ for two mintutes. The 
devices were baked to melt the wax, which allowed it to diffuse through the cellulosic matrix of the paper substrate and form the hydrophobic barriers.

In addition to chromatography paper, 3D devices were made out of modified paper, nitrocellulose, and printer paper panels and were fabricated using the same technique. The modified paper was produced by reacting the paper with $0.104 \mathrm{M}$ sodium periodate for 24 hours.

All the devices were prepared by depositing $1 \mu \mathrm{L}$ of the polymer solution either in the middle of the channel for the lateral flow devices or in the outer wells of the middle panel for the vertical flow devices unless noted otherwise. The lateral flow devices were dried for at least 30 minutes at room temperature after deposition of the polymer solution, and the vertical flow devices were dried for at least 1 hour at room temperature after deposition of the polymer solution unless noted otherwise. For the nitrocellulose panels, devices were printed and baked at $125^{\circ} \mathrm{C}$ for 5 minutes. ${ }^{13}$ The printer paper panels were baked according to the standard procedure.

Chromatography paper was modified by reacting select device panels, the polymer panels, with sodium periodate ${ }^{11}$, which oxidized the free hydroxyl groups in the cellulose. For the modified device panels, a fabricated panel was placed in a plastic petri dish and covered with $\sim 10 \mathrm{~mL}$ of a $0.104 \mathrm{M}$ sodium periodate solution. The petri dish was sealed with Parafilm to prevent the solution from leaking and was wrapped with aluminum foil. The panel was allowed to react for 24 hours before being removed from the solution, rinsed with NanoPure water, and dried between paper towels under a large 
book to draw out moisture. The panel was then prepared in the same way as the other panels.

\subsubsection{Lateral Flow Devices}

The first lateral flow device design used for this research was the modified version of the bone device depicted in Figure 8. The device had a central inlet zone with two channels branching off to either side. One channel had the polymer deposited in the center for the results zone, and the other channel was left blank as a control. For these devices, the device time was calculated by subtracting the time the sample took to reach the control zone from the time it took to reach the results zone $(\Delta t)$. For the regular bone devices and the 3D vertical flow devices, the device time was simply the amount of time that it took the sample to wick from the sample well through to the results well.

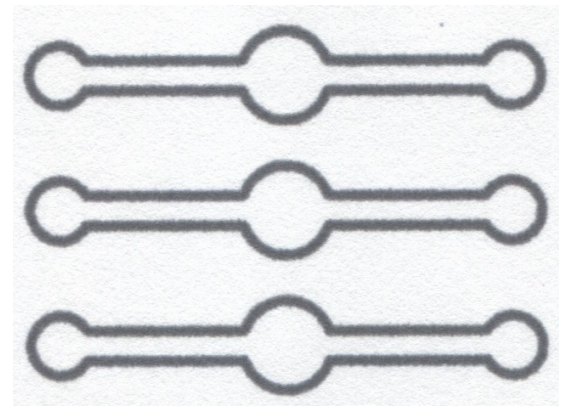

Figure 8: Modified Bone Devices

A normal unmodified bone device, depicted in Figure 2, was also used during the last round of testing with the final aqueous solutions. One well served as the inlet zone where the sample was deposited, the polymer was deposited in the channel, and the other well acted as the results zone so that the device time could be determined. For the initial device testing, a completed device was defined as a device wherein the results zone was 
completely filled in with the sample. This definition was later modified such that a device was considered finished once the sample reached the end of the channel.

\subsubsection{Vertical Flow Devices}

3D vertical flow devices were printed and fabricated as strips of panels or layers as denoted in Figure 9.

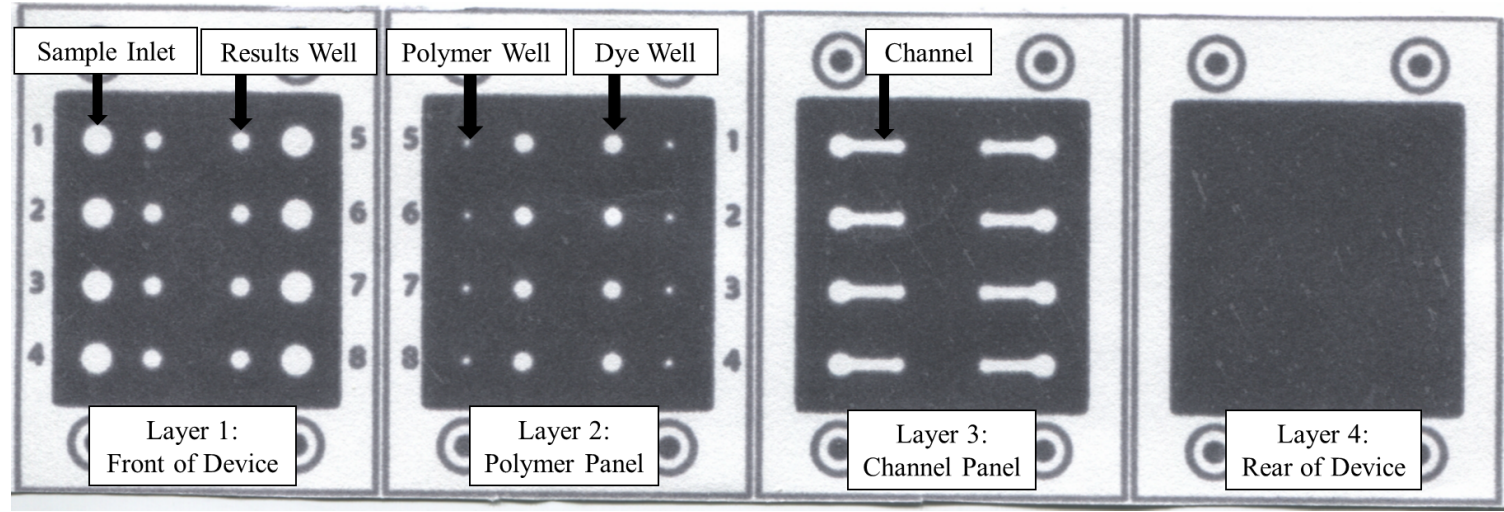

Figure 9: 3D Device Strip

8 devices on one fabricated strip

After fabrication, the devices were folded accordion style and sandwiched between two clear plastic plates held together by nuts and bolts that were hand tightened to the maximum torque as denoted in Figure 10.

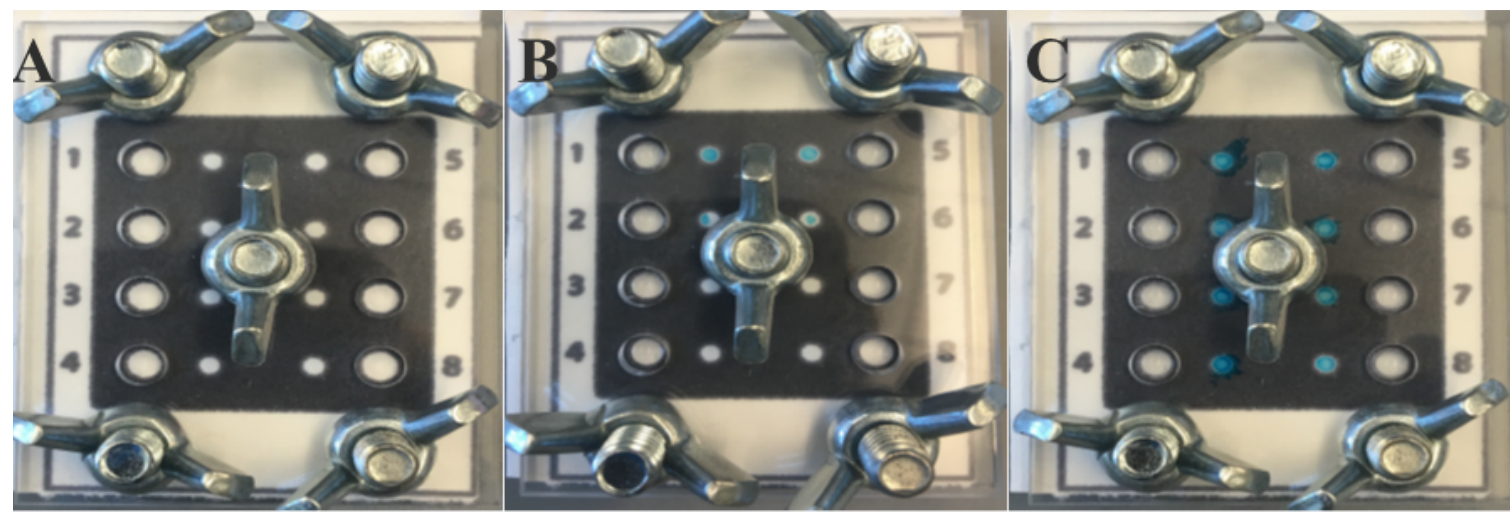

Figure 10: Assembled 3D Devices with no Polymer Deposited A - Before Sample Deposition, B - After Sample Deposition, and C - Set of Finished Devices 
The sample was deposited in the sample inlet well and, for most of the devices, immediately encountered the polymer layer. Once the sample wicked through the polymer layer, it wicked down to and across the channel in the third layer. After crossing the channel, the sample flowed up through the top two layers and transported a dye from the middle layer into the results well. A diagram of the layered device panels as well as the path followed by the sample is noted in Figure 11.

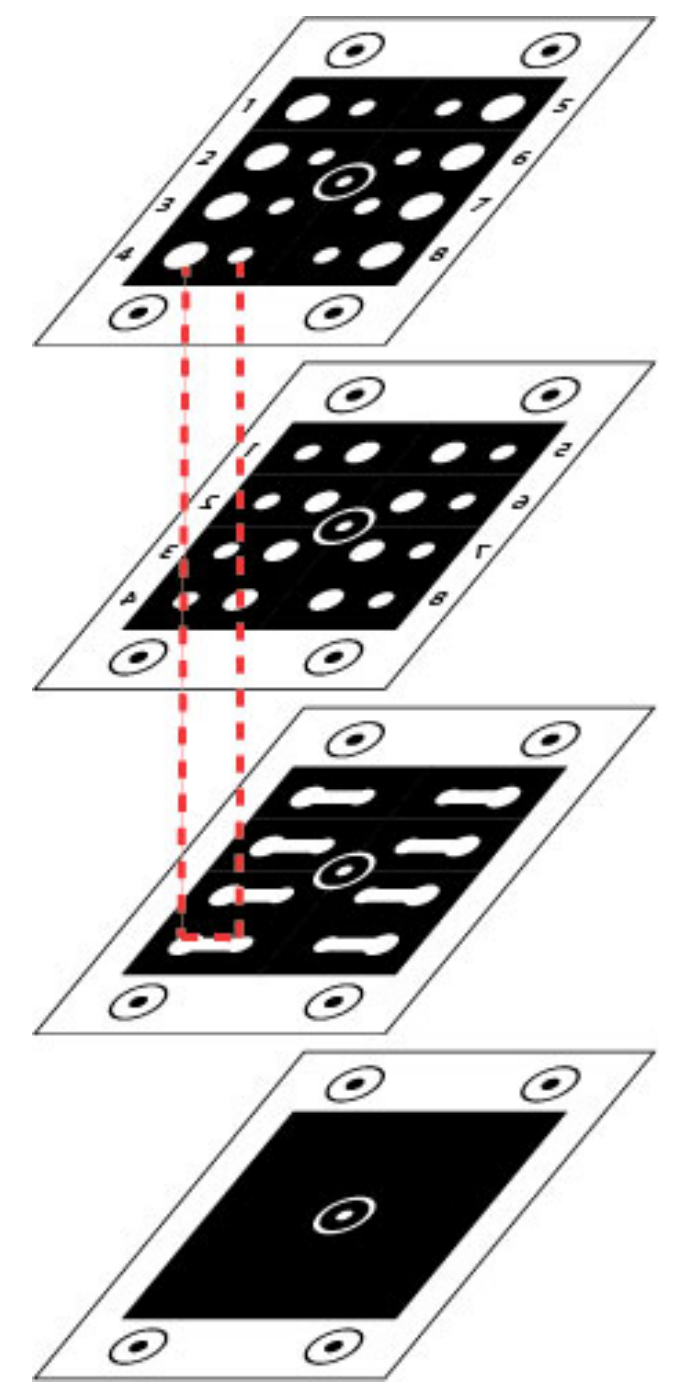

Figure 11: Example 3D Device with Sample Flow traced through Device 
For the initial device tests, the sample was considered to have finished when the results well completely filled with either dye or sample. This definition was later amended such that a device was considered to have finished when the results well began to fill with either dye or sample. For some of the device tests, the second layer of the device was substituted for modified paper, nitrocellulose, or printer paper.

Two different methods to wash the devices after drying the polymer were also investigated. The ultimate goal of this strategy was to determine an effective device preparation that could maintain the trend noted in other tests while reducing the standard deviation for devices. The first wash method involved adding $20 \mu \mathrm{L}$ of either NanoPure water or $0.005 \mathrm{M} \mathrm{HCl}$ to the device after it was assembled. After the solution wicked through the device to the results well, the strip of devices was then disassembled and dried at room temperature for 1 hour before being reassembled and tested. Alternatively, for the second wash method, the second layer of the device was placed in a plastic Petri dish with enough NanoPure water to cover the panel. The panels were stirred (Lab-Line 3D Rotator) for either 1 minute or 20 minutes. The devices were patted dry with a paper towel and were allowed to dry completely at room temperature for 1 hour. 


\section{RESULTS AND DISCUSSION}

\subsection{Polymer Synthesis and Characterization}

The initial sample of PAPBA used for this research project was synthesized by the Sumerlin group at the University of Florida. The molecular weight distribution determined via GPC is given in Table 2. The moderate molecular weight of the polymer coincides with what would be expected for a RAFT polymerization. However, the PDI value is a little larger than expected. Typically, RAFT polymerization yields polymers with a PDI close to one. One possible explanation for the larger-than-expected PDI is that the polymerization might be limited based on the reactivity of the side chain functional groups and the need for protecting groups for the boronic acid during the polymerization to prevent premature reactions.

Table 2: Polymer Molecular Weight Distribution for the Initial PAPBA sample

\begin{tabular}{|c|c|}
\hline $\mathbf{M}_{\mathbf{n}}$ & $13,350 \mathrm{~g} / \mathrm{mol}$ \\
\hline $\mathbf{M}_{\mathbf{w}}$ & $22,330 \mathrm{~g} / \mathrm{mol}$ \\
\hline PDI & 1.673 \\
\hline
\end{tabular}

\subsection{Polymer Solution Preparation and Characterization}

The PAPBA sample was first dissolved in basic solution $(\sim 0.1 \mathrm{M} \mathrm{NaOH})$. The stock solution was prepared with an approximate concentration of $12 \% \mathrm{w} / \mathrm{w}$. The exact concentration of this initial stock polymer solution (ISPS) was not known because of an error with the tare mechanism for the balance. The error was not noticed until after the initial aqueous solution had been already produced. The decision was made to begin 
testing with this solution and to simply approximate the concentration as closely as possible. This mistake was noted for future solutions and was not repeated for the methanol and the final aqueous solutions.

In addition to the problems with the analytical balance, we expected that the polymer would dissolve in water with a $\mathrm{pH}$ above 8.5 , but it required a much higher $\mathrm{pH}$ than expected, which necessitated multiple adjustments with increasingly more concentrated Sodium Hydroxide solutions. Once the solution was prepared, it was diluted and analyzed via DLS with the goal of using the data to determine the particle size distribution based on the polymer concentration. However, the noted intensity for each solution unfortunately does not correlate exactly to the concentration for that particular particle size. Based on previously published work on polymeric materials using DLS analysis, the average particle size for the PBBA sample based off the molecular weight was theoretically determined to be around $5 \mathrm{~nm} .^{14}$

Results from the analysis of the particle size distribution of solutions of the original PAPBA sample in $\sim 0.1 \mathrm{M} \mathrm{NaOH}$ are shown in Figures $8 \mathrm{a}-\mathrm{d}$. The particle size distribution based off the given intensity for the SD1 shows three clusters of particles with average radii of $5 \mathrm{~nm}, 40 \mathrm{~nm}$, and $200 \mathrm{~nm}$ (Figure 8a). When the solution was diluted to SD2, the cluster of particles with an average radius of $200 \mathrm{~nm}$ disappeared from the curve (Figure $8 \mathrm{~b}$ ). One possible explanation is that the larger particles observed in the $\sim 12 \% \mathrm{w} / \mathrm{w}$ solution are actually aggregates of smaller particles, and, as the polymer was diluted, the larger particle clusters de-aggregated to produce smaller particles. However, the larger particles and aggregates might make up a much smaller portion of the solution than that which is denoted on the graph. The presence of a few large particles 
within a solution can skew the DLS results as the large particles cause a large amount of scattering due to their size, which can interfere with the scattering for the smaller particles. This interference increases the intensity for the larger particles relative to the small particles giving the impression that there is a higher portion of these much larger particle sizes within the solution than is actually present.

As the polymer was diluted further to SD3 and SD4, the relative intensity of 5-nm particles increased, and the relative intensity of 50-nm particles decreased (Figures 8c-d). This result further suggests either that the individual polymer particles have a radius of 5$\mathrm{nm}$ and that these aggregate at higher concentrations to form the larger particles, or that the dilution reduces the overall effect of the larger particle sizes by minimizing the scattering interference introduced by the larger particles. It is interesting to note that particle aggregates of a particular (e.g. $50 \mathrm{~nm}, 200 \mathrm{~nm}$ ) size appear to be more favored than others.
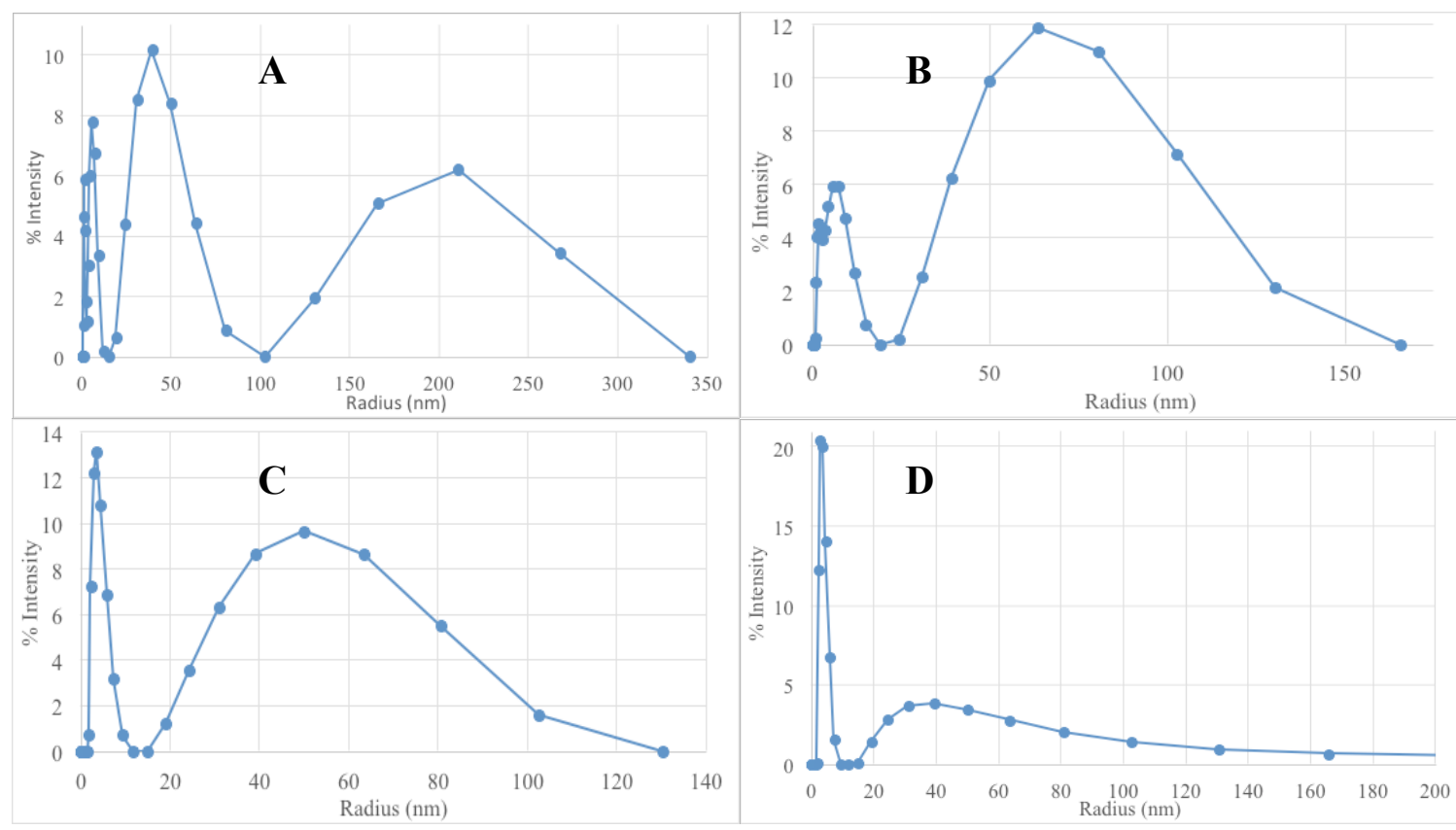

Figure 12: DLS Results for Four SD Solutions $\mathrm{A}-\mathrm{SD} 1, \mathrm{~B}-\mathrm{SD} 2, \mathrm{C}-\mathrm{SD} 3$, and D - SD4 
The solution of PBBA in methanol was much easier to prepare as the polymer dissolved readily in the less polar solvent. Solutions of PBBA in mixtures of methanol and water were also explored. It was determined that the polymer was soluble in an 80:20 methanol:water mixture up to a concentration of $4.685 \% \mathrm{w} / \mathrm{w}$. If the water content was increased above this ratio, then the polymer would precipitate out of the solution.

\subsection{Initial Aqueous Solution Tests}

\subsubsection{Lateral Flow Devices}

The volume of sample required to fill the modified bone device was first determined to be $30 \mu \mathrm{L}$. Then the $\sim 12 \% \mathrm{w} / \mathrm{w}$ ISPS was deposited in the middle of the channel of the device and dried. Alternatively, for some of the devices, a $2 \mu \mathrm{L}$ aliquot of $0.5 \mathrm{M} \mathrm{HCl}$ was deposited first into the middle of the channel followed by the polymer solution which was deposited on top. The hypothesis was that the acid would lower the $\mathrm{pH}$ of the polymer solution causing it to precipitate out of the solution and form a barrier within the cellulosic matrix of the channel. The results are shown in Table 3 and include the time it took for the sample to wick from the sample inlet across the channel and into the test zone. 
Table 3: Initial Glucose Tests with ISPS $(n=3)$

* Devices actually finished several minutes after the time was stopped though the time was not noted.

\begin{tabular}{|c|c|c|c|}
\hline Sample & Avg. $\Delta$ t (s) & StDev. (s) & \% Failed \\
\hline $\begin{array}{c}50.5 \text { mM Glucose } \\
\text { in NanoPure water }\end{array}$ & 368 & 25 & 0 \\
\hline $\begin{array}{c}25.3 \text { mM Glucose } \\
\text { in NanoPure water }\end{array}$ & 383 & 101 & 0 \\
\hline $\begin{array}{c}\text { NanoPure water } \\
\text { in 1XPBS }\end{array}$ & 754 & 16 & 33 \\
\hline $\begin{array}{c}54.1 \text { mM Glucose } \\
\text { in 1XPBS }\end{array}$ & 330 & 58 & 0 \\
\hline $\begin{array}{c}\text { 1XPBS } \\
\text { mM Glucose }\end{array}$ & N/A & N/A & $100^{*}$ \\
\hline
\end{tabular}

As expected, all the samples containing glucose wicked across the channel in less time than the samples that did not contain glucose (e.g., Nanopure water and 1XPBS). However, there were no statistically significant differences in the wicking times for any of the concentrations of glucose that were tested. One potential explanation for this observation is that the concentrations of glucose that were tested were outside the effective range of the assay. Another interesting observation is that the NanoPure water wicked across the channel faster than 1 XPBS, but the glucose solutions in NanoPure water appear to wick more slower than the glucose solutions in 1XPBS. While these experiments were performed, it was noted that solid polymer was still present within the ISPS meaning that the polymer was not completely dissolved within the solution. So, the polymer mixture was further diluted with $1 \mathrm{M} \mathrm{NaOH}$ in order to completely dissolve the polymer and make the stock polymer solution (SPS). The SPS was tested using two 
different deposition methods, with and without added acid. The results for this test are shown in Table 4.

Table 4: Deposition Method Tests with SPS (n=3)

\begin{tabular}{|c|c|c|c|c|}
\hline Polymer Solution & Sample & Avg. $\Delta t(s)$ & StDev. (s) & \% Failed \\
\hline Plain SPS & \multirow{2}{*}{$\begin{array}{c}\text { NanoPure } \\
\text { water }\end{array}$} & 23 & 17 & 0 \\
\hline Acid SPS & & 86 & 35 & 0 \\
\hline
\end{tabular}

Nanopure water wicked across both channels very rapidly compared to the more concentrated polymer solution, the ISPS. This was expected because a smaller amount of polymer in the channel should have less of an effect on wicking. Deposition of the polymer with acid had a longer wicking time. One possible explanation for this result was that the sudden change in the $\mathrm{pH}$ induced by the addition of a strong acid caused the polymer to precipitate out of solution on the device. However, based on these results, the plain polymer deposition method was tested further due to the lower standard deviation as this seemed to indicate less variability between devices. The SPS was tested with two 1XPBS-Glucose solutions (27.1 mM and $13.53 \mathrm{mM})$ as well, and both solutions failed wick across the channel. As a result, the flow rate was found to be too slow for practical diagnostic applications.

A final stock polymer solution (FSPS) was then prepared by diluting the remaining suspended polymer solution with $0.1 \mathrm{M} \mathrm{NaOH}$ and tested using the two different deposition methods. The results from this test are shown in Table 5. 
Table 5: Deposition Method Tests with FSPS (n=8)

\begin{tabular}{|c|c|c|c|c|}
\hline Polymer Solution & Sample & Avg. $\Delta$ t (s) & StDev. (s) & \% Failed \\
\hline Acid FSPS & \multirow{2}{*}{$\begin{array}{c}\text { NanoPure } \\
\text { water }\end{array}$} & 32 & 14 & 13 \\
\cline { 3 - 5 } Plain FSPS & N/A & N/A & 100 \\
\hline
\end{tabular}

Based on these results, the plain polymer deposition method was determined to be the ideal method because the acid treatment seemed to shorten the analysis time for a blank sample. The results for this deposition method were consistently too fast to be used for practical clinical applications so the acid deposition method was discarded. As a result, the plain polymer deposition method was used for the rest of the testing performed unless noted otherwise. A second set of tests with FSPS was performed. Results are noted in Table 6.

Table 6: Glucose Tests with FSPS using the Plain Polymer Deposition $(\mathrm{n}=3)$

\begin{tabular}{|c|c|c|c|}
\hline Sample & Avg. $\Delta$ t (s) & StDev. (s) & \% Failed \\
\hline NanoPure water & N/A & N/A & 100 \\
\hline 1XPBS & N/A & N/A & 100 \\
\hline $\begin{array}{c}27.1 \mathrm{mM} \text { Glucose } \\
\text { in 1XPBS }\end{array}$ & 1196 & 30 & 0 \\
\hline
\end{tabular}

The reason the 1XPBS and NanoPure water devices failed to finish was, in theory, because the polymer was too concentrated on the device. Thus, serial dilutions of FSPS were prepared using $0.1 \mathrm{M} \mathrm{NaOH}$. All the serial dilutions were tested with the same samples to determine if any of the serial dilutions were an improvement. 
All the SD1 devices failed to finish. For SD2, the NanoPure water and 1XPBS devices failed to finish, and the glucose devices took $\sim 30$ minutes before the sample actually finished. The results for the SD3 tests are denoted below in Table 7.

Table 7: Initial Glucose Tests with $\operatorname{SD3}(n=3)$

\begin{tabular}{|c|c|c|c|}
\hline Sample & Avg. $\Delta t$ (s) & StDev. (s) & \% Failed \\
\hline NanoPure water & N/A & N/A & 100 \\
\hline 1XPBS & 922 & 72 & 0 \\
\hline $\begin{array}{c}27.1 \text { mM Glucose } \\
\text { in 1XPBS }\end{array}$ & 630 & 60 & 0 \\
\hline
\end{tabular}

Based on the results, the research seemed to confirm the hypothesis that the stock polymer solutions were too concentrated for the detection of glucose. The SD3 tests were repeated, and SD4 was tested as well. The results are denoted in Table 8.

Table 8: Glucose Tests with SD3 and SD4 $(n=3)$

*Device mount was disturbed causing two devices to fail to finish.

\begin{tabular}{|c|c|c|c|c|}
\hline Polymer Solution & Sample & Avg. $\Delta$ t (s) & StDev. (s) & \% Failed \\
\hline \multirow{4}{*}{ SD4 } & NanoPure water & 298 & N/A & $67^{*}$ \\
\cline { 2 - 5 } & 1XPBS & 134 & 18 & 0 \\
\cline { 2 - 5 } & $\begin{array}{c}27.1 \text { mM Glucose } \\
\text { in 1XPBS }\end{array}$ & 124 & 10 & 0 \\
\hline \multirow{4}{*}{ SD3 } & NanoPure water & 1744 & 194 & 0 \\
\cline { 2 - 5 } & 1XPBS & 903 & 172 & 0 \\
\cline { 2 - 5 } & $\begin{array}{c}27.1 \text { mM Glucose } \\
\text { in 1XPBS }\end{array}$ & 845 & 135 & 0 \\
\hline
\end{tabular}


The SD4 devices have a low standard deviation, but there is no longer a trend dependent on the glucose concentration for these devices. Thus, it was hypothesized that with SD4 not enough polymer was now being deposited onto the devices, and, as a result, the devices were finishing too quickly because the sample could easily wick across the polymer. On the other hand, SD3 devices had a good trend based on the glucose concentration of the sample, but the large standard deviations meant that the results were not statistically significant. This ideal concentration was hypothesized to be somewhere in between that of SD2 and SD3 so they were tested again. The results for these tests are denoted below in Table 9.

Table 9: SD2 and SD3 Results $(n=6)$

\begin{tabular}{|c|c|c|c|c|}
\hline Polymer Solution & Sample & Avg. $\Delta$ t (s) & StDev. (s) & \% Failed \\
\hline \multirow{2}{*}{ SD2 } & NanoPure water & 3213 & N/A & 83 \\
\cline { 2 - 5 } & 1 1XPBS & 474 & 182 & 50 \\
\cline { 2 - 5 } & $\begin{array}{c}27.1 \text { mM Glucose } \\
\text { in 1XPBS }\end{array}$ & 319 & 150 & 0 \\
\hline \multirow{2}{*}{ SD3 } & \begin{tabular}{c} 
NanoPure water \\
\cline { 2 - 5 }
\end{tabular} & 861 & 107 & 50 \\
\cline { 2 - 5 } & $\begin{array}{c}\text { 1XPBS } \\
27.1 \text { mM Glucose } \\
\text { in 1XPBS }\end{array}$ & 453 & 79 & 0 \\
\hline
\end{tabular}

The results for both solutions were not promising as both SD2 and SD3 had large standard deviations that made any noted trends statistically insignificant. This result was, in part, expected for the SD2 devices especially given that all the previous SD2 devices failed to finish, but this result was surprising for SD3 given the previous tests. After these 
tests, it was hypothesized that there might be some residual solvent left in the polymer layer after drying. To test this hypothesis, the focus of the research project shifted to experiments that could determine what effect the drying time had on the reactivity of the PBBA. The tests were therefore repeated for SD2 with drying times of 30 minutes, 2 hours, 4 hours, and 24 hours at room temperature as noted in Table 10.

Table 10: Effect of Drying Time on Devices for SD2 $(n=3)$

\begin{tabular}{|c|c|c|c|c|}
\hline Drying Time & Sample & Avg. $\Delta t(s)$ & StDev. (s) & $\%$ Failed \\
\hline \multirow{3}{*}{30 Minutes } & NanoPure water & 4180 & 15 & 33 \\
\hline & 1XPBS & N/A & N/A & 100 \\
\hline & $\begin{array}{l}27.1 \mathrm{mM} \text { Glucose } \\
\text { in } 1 \mathrm{XPBS}\end{array}$ & 2724 & 317 & 0 \\
\hline \multirow{3}{*}{2 Hours } & NanoPure water & N/A & N/A & 100 \\
\hline & 1XPBS & N/A & N/A & 100 \\
\hline & $\begin{array}{l}27.1 \mathrm{mM} \text { Glucose } \\
\text { in 1XPBS }\end{array}$ & 4058 & 1275 & 33 \\
\hline \multirow{3}{*}{4 Hours } & NanoPure water & N/A & N/A & 100 \\
\hline & 1XPBS & N/A & N/A & 100 \\
\hline & $\begin{array}{l}27.1 \mathrm{mM} \text { Glucose } \\
\text { in } 1 \text { XPBS }\end{array}$ & N/A & N/A & 100 \\
\hline \multirow{3}{*}{24 Hours } & NanoPure water & 1948 & N/A & 67 \\
\hline & 1XPBS & 1115 & 285 & 0 \\
\hline & $\begin{array}{l}27.1 \mathrm{mM} \text { Glucose } \\
\text { in 1XPBS }\end{array}$ & 655 & 158 & 0 \\
\hline
\end{tabular}

While most of the results display a large amount of variability due to the sizable standard deviations for those devices that did finish, the 24 hour devices seemed to 
demonstrate the glucose based trend that was the target for this project; thus, the dry time experiments were investigated further using SD2. The following drying times were investigated: 30 minutes, 24 hours, and 48 hours, and the following drying temperatures were investigated as well: $50{ }^{\circ} \mathrm{C}$ and $100^{\circ} \mathrm{C}$. The results are noted in Table 11 .

Table 11: Effect of Drying Time and Temperature for SD2 (n=3)

\begin{tabular}{|c|c|c|c|c|}
\hline Drying Conditions & Sample & Avg. $\Delta t(s)$ & StDev. (s) & $\%$ Failed \\
\hline \multirow{3}{*}{30 Minutes } & NanoPure water & 3794 & 70.71 & 33 \\
\hline & 1XPBS & 4260 & N/A & 67 \\
\hline & $\begin{array}{l}27.1 \mathrm{mM} \text { Glucose } \\
\text { in 1XPBS }\end{array}$ & N/A & N/A & 100 \\
\hline \multirow{3}{*}{24 Hours } & NanoPure water & N/A & N/A & 100 \\
\hline & 1XPBS & N/A & N/A & 100 \\
\hline & $\begin{array}{l}27.1 \mathrm{mM} \text { Glucose } \\
\text { in } 1 \text { XPBS }\end{array}$ & N/A & N/A & 100 \\
\hline \multirow{3}{*}{48 Hours } & NanoPure water & 3498 & N/A & 67 \\
\hline & 1XPBS & 1880 & 551 & 0 \\
\hline & $\begin{array}{l}27.1 \mathrm{mM} \text { Glucose } \\
\text { in 1XPBS }\end{array}$ & 1263 & 284 & 0 \\
\hline \multirow{3}{*}{$50{ }^{\circ} \mathrm{C}$} & NanoPure water & 1761 & 283 & 0 \\
\hline & 1XPBS & 1157 & 219 & 0 \\
\hline & $\begin{array}{l}27.1 \mathrm{mM} \text { Glucose } \\
\text { in 1XPBS }\end{array}$ & 789 & 54 & 0 \\
\hline \multirow{3}{*}{$100^{\circ} \mathrm{C}$} & NanoPure water & 2385 & 704 & 33 \\
\hline & 1XPBS & 2334 & 539 & 0 \\
\hline & $\begin{array}{l}27.1 \mathrm{mM} \text { Glucose } \\
\text { in 1XPBS }\end{array}$ & 658 & 83 & 0 \\
\hline
\end{tabular}


Based on the results that had been obtained so far for all the lateral flow devices, the decision was made to switch to using 3D vertical flow devices. While some general associations were detected using the lateral flow devices, a large amount of variability was present within and between the tests conducted. As such, the hypothesis was that the device type might adversely affect the reaction thus the research shifted to focus on using vertical flow devices.

\subsubsection{Vertical Flow Devices}

SD1 was tested first on the vertical flow devices using 1XPBS and one 1XPBSGlucose solution $(27.1 \mathrm{mM})$. The results from the tests are denoted in Table 12.

Table 12: SD1 Vertical Flow Test Results $(n=2)$

\begin{tabular}{|c|c|c|c|}
\hline Sample & Avg. t (s) & StDev. (s) & \% Failed \\
\hline 1XPBS & 1372 & 937 & 0 \\
\hline $\begin{array}{c}27.1 \mathrm{mM} \text { Glucose } \\
\text { in 1XPBS }\end{array}$ & N/A & N/A & 100 \\
\hline
\end{tabular}

Because of how extreme the results were, the test with SD1 was performed again using a different 1XPBS-Glucose concentration $(13.53 \mathrm{mM})$. The results from these tests are denoted below in Table 13.

Table 13: SD1 Vertical Flow Test Results, Repeat Test $(n=2)$

\begin{tabular}{|c|c|c|c|}
\hline Sample & Avg. t (s) & StDev. (s) & \% Failed \\
\hline 1 XPBS & 804 & 19 & 0 \\
\hline $\begin{array}{c}13.53 \mathrm{mM} \text { Glucose } \\
\text { in 1XPBS }\end{array}$ & 1701 & N/A & 50 \\
\hline
\end{tabular}


Based on these results, SD2 and SD3 were both investigated next to determine the effect of the polymer solution on device times. These tests were run using the same samples as the previous test. However, almost all the devices failed to finish, and the one device that did finish did not finish until well after 30 minutes. SD1 was examined again though using a broader range of glucose concentrations this time. The tests were run using 1XPBS and three 1XPBS-Glucose solutions (13.53 mM, $6.76 \mathrm{mM}$, and $3.38 \mathrm{mM})$. The results are denoted below in Table 14 .

Table 14: SD1 with Range of Glucose Concentrations (n=2)

\begin{tabular}{|c|c|c|c|}
\hline Sample & Avg. t (s) & StDev. (s) & \% Failed \\
\hline 1XPBS & 2101 & 690 & 0 \\
\hline $\begin{array}{c}\text { 13.53 mM Glucose } \\
\text { in 1XPBS }\end{array}$ & N/A & N/A & 100 \\
\hline $\begin{array}{c}6.76 \mathrm{mM} \text { Glucose } \\
\text { in 1XPBS }\end{array}$ & 1160 & N/A & 50 \\
\hline $\begin{array}{c}3.38 \mathrm{mM} \text { Glucose } \\
\text { in 1XPBS }\end{array}$ & 1554 & 660 & 0 \\
\hline
\end{tabular}

Upon further analysis, the polymer wells were determined to be too small for the application due to the changes made in the fabrication procedure. The original device design was fabricated at a lower temperature for a longer period of time as compared to the procedure used for this research. It was hypothesized that the higher temperature for the new procedure might explain the variability between devices. The polymer wells were expanded on all sides by $1 \mathrm{~mm}$ to account for this possibility. SD1, SD2, and SD3 were tested with these enlarged polymer well devices using 1XPBS and one 1XPBSGlucose solution (13.53 mM). All but one of the devices failed to finish. Based off these 
results, another serial dilution was performed on SD4 yielding SD5. SD3, SD4, and SD5 were all tested using both the normal polymer wells and the enlarged polymer wells with both using the same samples as the previous tests. The results for the tests are denoted below in Table 15.

Table 15: Comparison of Normal Polymer Wells with Enlarged Polymer Wells (n=2)

\begin{tabular}{|c|c|c|c|c|c|}
\hline $\begin{array}{l}\text { Device } \\
\text { Type }\end{array}$ & $\begin{array}{l}\text { Polymer } \\
\text { Solution }\end{array}$ & Sample & Avg. t (s) & StDev. (s) & $\%$ Failed \\
\hline \multirow[b]{2}{*}{ Normal } & \multirow{4}{*}{ SD3 } & 1XPBS & N/A & N/A & 100 \\
\hline & & $\begin{array}{l}13.53 \mathrm{mM} \text { Glucose } \\
\text { in 1XPBS }\end{array}$ & N/A & N/A & 100 \\
\hline \multirow{2}{*}{ Enlarged } & & 1XPBS & N/A & N/A & 100 \\
\hline & & $\begin{array}{l}13.53 \mathrm{mM} \text { Glucose } \\
\text { in 1XPBS }\end{array}$ & 199 & 33 & 0 \\
\hline \multirow{2}{*}{ Normal } & \multirow{4}{*}{ SD4 } & 1XPBS & N/A & N/A & 100 \\
\hline & & $\begin{array}{l}13.53 \mathrm{mM} \text { Glucose } \\
\text { in 1XPBS }\end{array}$ & N/A & N/A & 100 \\
\hline \multirow[b]{2}{*}{ Enlarged } & & 1XPBS & 94 & 13 & 0 \\
\hline & & $\begin{array}{l}13.53 \mathrm{mM} \text { Glucose } \\
\text { in 1XPBS }\end{array}$ & 78 & 18 & 0 \\
\hline \multirow{2}{*}{ Normal } & \multirow{4}{*}{ SD5 } & 1XPBS & 479 & 251 & 0 \\
\hline & & $\begin{array}{l}13.53 \mathrm{mM} \text { Glucose } \\
\text { in 1XPBS }\end{array}$ & 206 & 132 & 0 \\
\hline \multirow{2}{*}{ Enlarged } & & 1XPBS & 44 & 1 & 0 \\
\hline & & $\begin{array}{l}13.53 \mathrm{mM} \text { Glucose } \\
\text { in 1XPBS }\end{array}$ & 48 & 16 & 0 \\
\hline
\end{tabular}


Based on these results, the enlarged polymer well devices were used in place of the normal polymer well devices for this research project from this point forward unless noted otherwise. From there, SD1 was tested using both 1XPBS and a range of 1XPBSGlucose solution concentrations (13.53 mM, $6.76 \mathrm{mM}$, and $3.38 \mathrm{mM})$. However, all of the devices failed to finish completely. As a result, SD2 was skipped in favor of SD3. The results for these tests are denoted below in Table 16.

Table 16: SD3 Test with Glucose Concentration Range $(n=2)$

\begin{tabular}{|c|c|c|c|}
\hline Sample & Avg. t (s) & StDev. (s) & \% Failed \\
\hline 1 XPBS & 2126 & 786 & 0 \\
\hline $\begin{array}{c}\text { 13.53 mM Glucose } \\
\text { in 1XPBS }\end{array}$ & 690 & N/A & 50 \\
\hline $\begin{array}{c}\text { 6.76 mM Glucose } \\
\text { in 1XPBS }\end{array}$ & 413 & N/A & 50 \\
\hline $\begin{array}{c}3.38 \text { mM Glucose } \\
\text { in 1XPBS }\end{array}$ & 535 & 205 & 0 \\
\hline
\end{tabular}

These results show no real trend present and still demonstrate large standard deviations for those tests where enough devices finished so that the standard deviation could in fact be calculated. Thus, SD4 was tested next in the same way and using the same samples. The results for these tests are denoted below in Table 17. 
Table 17: SD4 Test with Glucose Concentration Range (n=2)

\begin{tabular}{|c|c|c|c|}
\hline Sample & Avg. t (s) & StDev. (s) & \% Failed \\
\hline 1XPBS & 69 & 8 & 0 \\
\hline $\begin{array}{c}\text { 13.53 mM Glucose } \\
\text { in 1XPBS }\end{array}$ & 77 & 13 & 0 \\
\hline $\begin{array}{c}\text { 6.76 mM Glucose } \\
\text { in 1XPBS }\end{array}$ & 113 & 11 & 0 \\
\hline $\begin{array}{c}3.38 \text { mM Glucose } \\
\text { in 1XPBS }\end{array}$ & 114 & N/A & 50 \\
\hline
\end{tabular}

The results received unfortunately demonstrate no trend with a change in the glucose concentration though they do have small standard deviations. Based on these results, SD3 was tested with NanoPure water, $0.01 \mathrm{M} \mathrm{HCl}, 0.01 \mathrm{M} \mathrm{NaOH}$, and one NanoPure-Glucose solution $(12.65 \mathrm{mM})$. The results for these tests are noted in Table 18 .

Table 18: NanoPure water, Acid, Base, and Glucose Test Results $(n=4)$

\begin{tabular}{|c|c|c|c|}
\hline Sample & Avg. t (s) & StDev. (s) & \% Failed \\
\hline NanoPure water & N/A & N/A & 100 \\
\hline $0.01 \mathrm{M} \mathrm{HCl}$ & 61 & 19 & 0 \\
\hline $0.01 \mathrm{M} \mathrm{NaOH}$ & 176 & 19 & 0 \\
\hline $\begin{array}{c}12.65 \mathrm{mM} \mathrm{Glucose} \\
\text { in NanoPure water }\end{array}$ & 445 & 114 & 25 \\
\hline
\end{tabular}

For these tests interestingly enough, the acid finished before the base which was not expected given the $\mathrm{pH}$ responsiveness of the PBBA. The hypothesis for this was that the acid was reacting with the residual base that was still mixed with the polymer during 
deposition even after drying. To test this hypothesis, SD3 was tested again in the same though with the following samples: $0.0177 \mathrm{M} \mathrm{NaCl}, 0.005 \mathrm{M} \mathrm{HCl}, 0.005 \mathrm{M} \mathrm{NaOH}, 0.01$ $\mathrm{M}$ acetic acid, and $10.1 \mathrm{mM}$ glucose dissolved in $0.005 \mathrm{M} \mathrm{NaOH}$. The results for these tests are denoted in Table 19.

Table 19: Acid, Base, and Salt Test Results $(n=4)$

\begin{tabular}{|c|c|c|c|}
\hline Sample & Avg. t (s) & StDev. (s) & \% Failed \\
\hline $0.0177 \mathrm{M} \mathrm{NaCl}$ & 133 & 106 & 31 \\
\hline $0.005 \mathrm{M} \mathrm{HCl}$ & 116 & 93 & 7 \\
\hline $0.005 \mathrm{M} \mathrm{NaOH}$ & 159 & 85 & 8 \\
\hline $0.01 \mathrm{M} \mathrm{Acetic} \mathrm{Acid}$ & 107 & 65 & 30 \\
\hline $\begin{array}{c}10.1 \mathrm{mM} \mathrm{Glucose} \mathrm{in} \\
0.005 \mathrm{M} \mathrm{NaOH}\end{array}$ & 304 & 340 & 13 \\
\hline
\end{tabular}

Again, the acid finished before the base though in this case the result is no longer statistically significant due to how close the averages are together and the magnitude of the standard deviations. This result is likely due to the fact that the solutions are now at half the concentration that was used in the previous test. There was concern that the folding of the devices might be damaging the polymer layer so a strip of devices was prefolded prior to the application of SD3 and was tested using NanoPure water and three NanoPure-Glucose solutions (12.65 mM, 6.325 mM, and $3.163 \mathrm{mM})$. The results for this test are denoted below in Table 20. 
Table 20: Pre-Folded Devices with SD3 $(n=4)$

\begin{tabular}{|c|c|c|c|}
\hline Sample & Avg. t (s) & StDev. (s) & \% Failed \\
\hline NanoPure water & 148 & 19 & 0 \\
\hline $\begin{array}{l}\text { 12.65 mM Glucose } \\
\text { in NanoPure water }\end{array}$ & 131 & 26 & 0 \\
\hline $\begin{array}{c}\text { 6.325 mM Glucose } \\
\text { in NanoPure water }\end{array}$ & 108 & 39 & 0 \\
\hline $\begin{array}{c}\text { 3.163 mM Glucose } \\
\text { in NanoPure water }\end{array}$ & 118 & 12 & 0 \\
\hline
\end{tabular}

These devices did not demonstrate a noticeable trend though they did demonstrate lower standard deviations. Because of this, the pre-folded technique was tested with SD2 using the same samples as the previous test. The results for this test are denoted below in Table 21.

Table 21: Pre-Folded Devices with SD2 $(n=4)$

\begin{tabular}{|c|c|c|c|}
\hline Sample & Avg. t (s) & StDev. (s) & \% Failed \\
\hline NanoPure water & 1354 & 342 & 0 \\
\hline $\begin{array}{l}\text { 12.65 mM Glucose } \\
\text { in NanoPure water }\end{array}$ & 1459 & 224 & 25 \\
\hline $\begin{array}{l}6.235 \text { mM Glucose } \\
\text { in NanoPure water }\end{array}$ & 1441 & 319 & 25 \\
\hline $\begin{array}{l}\text { 3.163 mM Glucose } \\
\text { in NanoPure water }\end{array}$ & 1511 & N/A & 75 \\
\hline
\end{tabular}

These tests also demonstrated no statistically significant trend dependent on the Glucose concentration while also yielding large standard deviations. After these tests, I 
met with my collaborators in the Sumerlin groups at the University of Florida to determine new directions for the research project.

\subsection{Methanol Solution Tests}

The $5 \% \mathrm{w} / \mathrm{v}$ thermal responsive boronic acid block copolymer solution was tested initially with $10.1 \mathrm{mM}$ glucose in $0.005 \mathrm{M} \mathrm{NaOH}$. Four replicates were performed for the devices. The average device time for these tests was 195 seconds with a standard deviation of 66 seconds. These results seemed promising so the solution was tested with a range of glucose concentrations $(0.1 \mathrm{M}, 0.05 \mathrm{M}, 0.025 \mathrm{M}, 0.015 \mathrm{M}$, and $0.01 \mathrm{M})$ dissolved in PBS buffer solution and the blank PBS buffer. The results for these tests are denoted in Table 22.

Table 22: 5 \% w/v Block Copolymer Solution Results $(n=4)$

\begin{tabular}{|c|c|c|c|}
\hline Sample & Avg. t (s) & StDev. (s) & \% Failed \\
\hline $\begin{array}{c}\text { 0.1 M Glucose in } \\
\text { PBS buffer }\end{array}$ & 384 & 35 & 0 \\
\hline $\begin{array}{c}0.05 \text { M Glucose } \\
\text { in PBS buffer }\end{array}$ & 488 & 41 & 0 \\
\hline $\begin{array}{c}0.025 \text { M Glucose } \\
\text { in PBS buffer }\end{array}$ & 430 & 23 & 0 \\
\hline $\begin{array}{c}0.015 \text { M Glucose } \\
\text { in PBS buffer }\end{array}$ & 423 & 64 & 0 \\
\hline $\begin{array}{c}0.01 \text { M Glucose } \\
\text { in PBS buffer }\end{array}$ & 485 & 46 & 0 \\
\hline PBS Buffer & 626 & 89 & 0 \\
\hline
\end{tabular}


While these results do not demonstrate a definitive trend, the standard deviations for these tests were lower than previously obtained values for the aqueous based solutions. Thus, the decision was made to switch from aqueous based solutions to methanol-based solutions for the initial polymer sample. Results from the initial testing with the pure Methanol solutions are noted below in Table 23.

Table 23: $3.88 \%$ w/w Methanol Solution Devices (n=4)

\begin{tabular}{|c|c|c|c|}
\hline Sample & Avg. t (s) & StDev (s) & \% Failed \\
\hline NanoPure water Run 1 & 121 & 41 & 25 \\
\hline $\begin{array}{c}\text { NanoPure water Run 2 } \\
\begin{array}{c}10.1 \mathrm{mM} \mathrm{Glucose} \mathrm{in} \\
0.005 \mathrm{M} \mathrm{NaOH}\end{array}\end{array}$ & 156 & 52 & 50 \\
\hline $\begin{array}{c}13.53 \mathrm{mM} \text { Glucose in } \\
\text { 1XPBS }\end{array}$ & N/A & N/A & 75 \\
\hline
\end{tabular}

A stock solution was made by dissolving a sample of the polymer in HPLC Grade Methanol to form a $7.76 \% \mathrm{w} / \mathrm{w}$ solution that was tested on a strip of vertical flow devices with NanoPure water. All the NanoPure water devices failed to finish so a serial dilution was performed yielding a $3.88 \% \mathrm{w} / \mathrm{w}$ solution and was tested initially with NanoPure water. The NanoPure water sample for the serial dilution finished within what was considered to be a reasonable time although one of the devices for this sample did fail to finish. The dilution was then tested with $10.1 \mathrm{mM}$ glucose in $0.005 \mathrm{M} \mathrm{NaOH}$ and one 1XPBS-Glucose solution $(13.53 \mathrm{mM})$. Both solutions tested were thus slightly basic. As noted above, almost all of the devices for these solutions failed to finish, and the one 
device that did finish was within the time range for the previous NanoPure water samples.

Because of these results and the fact that the methanol would diffuse through the wax barrier, a NanoPure water dilution of the stock polymer solution was formulated so that the pure methanol solutions could be tested without solvent diffusion. Several different solutions of methanol and water were tested on a matrix of concentric circles of wax barriers, and the most ideal solution was determined to be an 80:20 solution by volume of methanol:water. The stock solution was modified with NanoPure water to reach this volume fraction yielding a $5.13 \% \mathrm{w} / \mathrm{w}$ polymer solution. This solution was tested with NanoPure water and with two 1XPBS-Glucose solutions (13.53 mM and $3.381 \mathrm{mM}$ ). Initial results seemed to be promising so the solution was tested again this time with pure 1XPBS and with two different 1XPBS-Glucose solutions $(27.1 \mathrm{mM}$ and $1.691 \mathrm{mM})$. The results for these tests are noted below in Table 24 .

Table 24: $5.13 \%$ w/w Polymer Solution Devices $(n=4)$

\begin{tabular}{|c|c|c|c|c|}
\hline Test & Sample & Avg. t (s) & StDev (s) & \% Failed \\
\hline \multirow{4}{*}{ Initial Tests } & \begin{tabular}{c} 
NanoPure water \\
\cline { 2 - 5 }
\end{tabular} & 81 & 14 & 0 \\
\cline { 2 - 5 } & $\begin{array}{c}3.38 \text { in 1XPBS } \\
\text { in 1XPBS }\end{array}$ & 228 & 147 & 0 \\
\hline \multirow{3}{*}{$\begin{array}{c}\text { Repeat } \\
\text { Tests }\end{array}$} & $\begin{array}{c}27.1 \mathrm{mM} \text { Glucose } \\
\text { in 1XPBS }\end{array}$ & 323 & 75 & 0 \\
\cline { 2 - 5 } & $\begin{array}{c}1.691 \mathrm{mM} \text { Glucose } \\
\text { in 1XPBS }\end{array}$ & 249 & 212 & 0 \\
\hline
\end{tabular}


While these tests did demonstrate a change in time to finish with a change in the glucose concentration of the sample, the standard deviations for several of these tests were pretty large which is certainly not an ideal situation for a proposed clinical diagnostic device. After testing, methanol was added to ensure that the polymer remained dissolved creating a $4.10 \% \mathrm{w} / \mathrm{w}$ polymer solution. The solution was tested with NanoPure water, 1XPBS, and two 1XPBS-Glucose solutions (27.1 mM and $1.691 \mathrm{mM})$. The results from these tests are noted in Table 25.

Table 25: $4.10 \%$ w/w Methanol Solution $(n=4)$

\begin{tabular}{|c|c|c|c|}
\hline Sample & Avg. t (s) & StDev (s) & \% Failed \\
\hline NanoPure water & 80 & 15 & 0 \\
\hline 1XPBS & 93 & 16 & 0 \\
\hline $27.1 \mathrm{mM}$ Glucose in 1XPBS & 83 & 11 & 0 \\
\hline $1.691 \mathrm{mM}$ Glucose in 1XPBS & 82 & 3 & 0 \\
\hline
\end{tabular}

While the standard deviations for this solution are reduced, there no longer exists a correlation between device time and glucose concentration. The hypothesized reason for this was that the solution was not concentrated enough, and, because of this, the sample was able to move through the polymer layer. The last test for this solution was repeated using double the amount $(2 \mu \mathrm{L})$ of polymer solution to test the effect of adding more PBBA. However, almost all of the devices failed to finish, and the one device that did finish, one $1.691 \mathrm{mM}$ glucose device, finished with a time of almost 17 minutes. 
A dilution of the $3.88 \% \mathrm{w} / \mathrm{w}$ pure methanol solution was made in water to bring the solution to the determined 80:20 methanol:water concentration. This dilution produced a solution with a concentration of $3.40 \% \mathrm{w} / \mathrm{w}$ polymer. Instead of testing with the vertical flow devices as with the previous tests, this solution was initially tested with an array of the modified lateral flow bone devices. However, for these tests, the deposition method used was varied to try to determine a better deposition method for the methanol-based solutions.

One pair of devices simply had the polymer solution deposited in the channel, another pair of devices had a $2 \mu \mathrm{L}$ aliquot of NanoPure water deposited on top of the polymer solution, and the final pair of devices had two $1 \mu \mathrm{L}$ deposited on either side of the polymer solution. The hypothesis for the different deposition methods was that the large influx of NanoPure water would dilute the methanol so much that the polymer would precipitate out of the solution while on the device. The results for these tests are denoted in Table 26.

Table 26: Lateral Flow Test for $3.40 \%$ w/w Polymer Solution (n=3)

\begin{tabular}{|c|c|c|c|}
\hline Polymer Solution & Avg. $\Delta$ t (s) & StDev. (s) & \% Failed \\
\hline Pure Polymer & 683 & 107 & 0 \\
\hline $\begin{array}{c}\text { NanoPure water } \\
\text { on top }\end{array}$ & 431 & 109 & 0 \\
\hline $\begin{array}{c}\text { NanoPure water } \\
\text { on either side }\end{array}$ & 516 & 118 & 0 \\
\hline
\end{tabular}

The devices produced an interesting spread of times demonstrating a time difference, albeit not statistically significant, between the various deposition methods 
tested. However, the standard deviations are still quite large. In addition to these lateral flow tests, a strip of vertical flow devices was tested with this polymer solution with NanoPure water as the sample as well. For these tests, the devices finished quickly as the calculated average time for the polymer devices (40.5 seconds) was just barely above those devices setup as blanks (22.50 seconds) with low standard deviations for both samples.

Based off these results, the solution was tested again on the vertical flow devices though this time with 1XPBS and two 1XPBS-Glucose solutions (27.1 mM and 1.691 $\mathrm{mM}$ ). However, almost all of the devices failed to finish, and the device that did finish took over 17 minutes suggesting that too much polymer was present which contrasts with the previous NanoPure water sample as those devices finished quickly.

As a result, a serial dilution of the $3.40 \% \mathrm{w} / \mathrm{w}$ solution was performed which yielded a polymer solution with a concentration of $1.61 \% \mathrm{w} / \mathrm{w}$ solution. This dilution was tested on both the lateral flow devices as well as the vertical flow devices. Half of the set of the lateral flow devices were prepared using the NanoPure water on top deposition method wherein a $2 \mu \mathrm{L}$ aliquot of NanoPure water was deposited on top of the polymer solution while the other set was prepared using an unmodified deposition method. The devices were tested with both NanoPure water and one NanoPure-Glucose solution (12.65 mM). The vertical flow tests were prepared using an unmodified procedure and were tested with 1XPBS and two 1XPBS-Glucose solutions $(27.1 \mathrm{mM}$ and $1.691 \mathrm{mM})$. The results for these tests are noted in Table 27 for both the Lateral Flow and the Vertical Flow tests. 
Table 27: Lateral $(n=3)$ and Vertical $(n=4)$ Flow Tests for $1.61 \%$ w/w Solution

\begin{tabular}{|c|c|c|c|c|}
\hline Polymer Solution & Sample & Avg. t (s) & StDev. (s) & \% Failed \\
\hline \multirow{2}{*}{ Pure Polymer } & NanoPure water & 390 & 46 & 0 \\
\cline { 2 - 5 } & $\begin{array}{c}12.65 \text { mM Glucose } \\
\text { in NanoPure water }\end{array}$ & 459 & 106 & 0 \\
\hline \multirow{2}{*}{$\begin{array}{c}\text { NanoPure water on } \\
\text { Top }\end{array}$} & \begin{tabular}{c} 
NanoPure water \\
\cline { 2 - 5 }
\end{tabular} & 225 & 103 & 0 \\
\hline \multirow{2}{*}{$\begin{array}{c}12.65 \text { mM Glucose } \\
\text { in NanoPure water }\end{array}$} & 201 & 69 & 0 \\
\hline \multirow{2}{*}{$\begin{array}{c}\text { Vertical Flow } \\
\text { Devices }\end{array}$} & $\begin{array}{c}1.691 \text { mM Glucose } \\
\text { in 1XPBS }\end{array}$ & 38 & 6 & 0 \\
\cline { 2 - 5 } & $\begin{array}{c}27.1 \text { mM Glucose } \\
\text { in 1XPBS }\end{array}$ & 47 & 14 & 0 \\
\hline
\end{tabular}

While the lateral flow tests demonstrated a trend, the large standard deviations rendered the results statistically insignificant. One hypothesis was that the modified bone device was affecting capillary flow for the sample. Thus, the tests were repeated using the same samples though with unmodified bone devices as denoted in Table 28 .

Table 28: Unmodified Bone Devices with $1.61 \%$ w/w Solution $(n=3)$

\begin{tabular}{|c|c|c|c|c|}
\hline Polymer Solution & Sample & Avg. t (s) & StDev. (s) & \% Failed \\
\hline \multirow{2}{*}{ Pure Polymer } & NanoPure water & 456 & 78 & 0 \\
\cline { 2 - 5 } & $\begin{array}{l}12.65 \text { mM Glucose } \\
\text { in NanoPure water }\end{array}$ & 576 & 116 & 0 \\
\hline \multirow{2}{*}{$\begin{array}{c}\text { NanoPure water on } \\
\text { Top }\end{array}$} & NanoPure water & 477 & 73 & 0 \\
\cline { 2 - 5 } & $\begin{array}{l}12.65 \text { mM Glucose } \\
\text { in NanoPure water }\end{array}$ & 375 & 22 & 0 \\
\hline
\end{tabular}


The next set of lateral flow device tests demonstrated a more pronounced trend though again the standard deviations were so large that the results were not statistically significant as well. The vertical flow devices, on the other hand, had a low standard deviation though no longer demonstrated any trend with regards to the glucose concentration. Based off these results, an experiment was designed to determine how the polymer solution was reacting with the paper after being dissolved by the glucose solution.

Three $10 \mu \mathrm{L}$ aliquots of the stock pure methanol solution were combined with 10 $\mu \mathrm{L}$ of NanoPure water in separate, tared microcentrifuge tubes. Each aliquot had $5 \mu \mathrm{L}$ of either NanoPure water, the $6.325 \mathrm{mM}$ glucose solution, or the $12.65 \mathrm{mM}$ solution. These solutions were allowed to equilibrate for 30 minutes in their tubes before they were tested on individual lateral flow devices that were fabricated with longer channels lengths. All the devices failed to finish though the samples did make it partially through the channel, and the polymer solutions appeared to be a milky white suggesting that the polymer was only suspended in the solution and was not in fact completely dissolved explaining why the devices failed to finish.

Final testing focused on the effect of drying temperature on the polymer reactivity as it was hypothesized that some solvent might still be present within the polymer even after 30 minutes of drying. Thus, two strips of vertical flow devices were prepared with the $3.40 \% \mathrm{w} / \mathrm{w}$ polymer solution with one strip dried at room temperature for 30 minutes and the other strip dried at $60{ }^{\circ} \mathrm{C}$ for 30 minutes. The room temperature strip of devices was tested using $0.01 \mathrm{M} \mathrm{NaOH}$ and $0.01 \mathrm{M} \mathrm{HCl}$. The $60^{\circ} \mathrm{C}$ strip of devices was tested 
with two 1XPBS-Glucose solutions $(27.1 \mathrm{mM}$ and $1.691 \mathrm{mM})$. The results for these tests are denoted below in Table 29.

Table 29: Effect of Drying Temperature on PBBA Reactivity, Test $1(n=4)$

\begin{tabular}{|c|c|c|c|c|}
\hline Drying Conditions & Sample & Avg. t (s) & StDev. (s) & \% Failed \\
\hline Dried at $60^{\circ} \mathrm{C}$ & $\begin{array}{c}27.1 \mathrm{mM} \text { Glucose } \\
\text { in } 1 \mathrm{XPBS}\end{array}$ & N/A & N/A & 100 \\
\cline { 2 - 5 } & $\begin{array}{c}1.691 \mathrm{mM} \mathrm{Glucose} \\
\text { in } 1 \mathrm{XPBS}\end{array}$ & 1002 & 465 & 50 \\
\hline \multirow{2}{*}{ Room Temperature } & $0.01 \mathrm{M} \mathrm{NaOH}$ & 288 & 132 & 0 \\
\cline { 2 - 5 } & $0.01 \mathrm{M} \mathrm{HCl}$ & 376 & 334 & 0 \\
\hline
\end{tabular}

An increase in the drying time increased the standard deviation greatly for the devices that even managed to finish. At this point in the research then, the methanol solutions were not yielding any reproducible results that could be expanded from. Thus, one last strip of vertical flow tests were performed that were dried at $60{ }^{\circ} \mathrm{C}$ for 30 minutes with two 1XPBS-Glucose solutions $(27.1 \mathrm{mM}$ and $1.691 \mathrm{mM})$. The results for these tests are denoted below in Table 30.

Table 30: Effect of Drying Temperature on PBBA Reactivity, Test $2(n=4)$

\begin{tabular}{|c|c|c|c|c|}
\hline $\begin{array}{c}\text { Drying } \\
\text { Temperature }\end{array}$ & Sample & Avg. t (s) & StDev. (s) & \% Failed \\
\hline \multirow{2}{*}{$60^{\circ} \mathrm{C}$} & $\begin{array}{c}27.1 \mathrm{mM} \text { Glucose } \\
\text { in 1XPBS }\end{array}$ & 363 & 346 & 50 \\
\cline { 2 - 5 } & $\begin{array}{c}1.691 \mathrm{mM} \text { Glucose } \\
\text { in 1XPBS }\end{array}$ & 68 & 38 & 25 \\
\hline
\end{tabular}


Based off these results, it was decided to move back to aqueous based polymer solutions as the effect of the methanol on the wax barrier is still unknown and might explain the lack reproducible results obtained from the tests. The hypothesis is that the methanol was dissolving some of the wax barrier increasing the porosity of the barrier as well as bringing some of the wax into the sample zone. This would explain why either the tests had low standard deviations and no trend or high standard deviations with a noticeable trend. For the first tests, the barrier was rendered porous enough that the sample could pass around the polymer barrier layer. However, if enough polymer was present, it would unevenly cover the channel resulting in a wide range of device times for the solution.

\subsection{Final Aqueous Solution Tests}

Another aqueous stock polymer solution was formulated with a concentration of $4.685 \%$ w/w polymer. After formulation, the solution was tested first with NanoPure water and then with two NanoPure-Glucose solutions (12.65 mM and $6.325 \mathrm{mM})$. The results for these tests are denoted in Table 31 .

Table 31: $4.685 \%$ w/w Solution Results $(n=4)$

\begin{tabular}{|c|c|c|c|}
\hline Sample & Avg. t (s) & StDev. (s) & \% Failed \\
\hline NanoPure water & 123 & 33 & 0 \\
\hline $\begin{array}{l}12.65 \text { mM Glucose } \\
\text { in NanoPure water }\end{array}$ & 90 & 23 & 0 \\
\hline $\begin{array}{l}6.325 \text { mM Glucose } \\
\text { in NanoPure water }\end{array}$ & 139 & 68 & 25 \\
\hline
\end{tabular}


While there seems to be a trend present with the two glucose samples, the standard deviations unfortunately mean that the results are not statistically significant. As a result of these tests, the devices were washed after drying to try to remove any residual base left within the polymer matrix that might be affecting the reaction.

The wash through method was tested first using the $4.685 \% \mathrm{w} / \mathrm{w}$ polymer solution and was tested with both NanoPure water and one NanoPure-Glucose solution (12.65 mM). While the wash through step was successful with all devices finishing, the devices all failed when exposed to the sample after being washed. However, for many of the devices, the sample managed to make it through the polymer layer to the channel below before being stopped. This result was intriguing as the devices that had failed to finish before had been halted by the polymer failing to make through to the channel at all.

The devices were also tested using the soak method with NanoPure water and one NanoPure-Glucose solution $(12.65 \mathrm{mM})$. The results for these tests are noted in Table 32.

Table 32: Soak Method Results for $4.685 \%$ w/w Polymer Solution (n=4)

\begin{tabular}{|c|c|c|c|c|}
\hline Drying Time & Sample & Avg. t (s) & StDev. (s) & \% Failed \\
\hline \multirow{2}{*}{$\begin{array}{c}\text { 0 Minutes } \\
\text { (Control) }\end{array}$} & \begin{tabular}{c} 
NanoPure water \\
12.65 mM Glucose \\
\cline { 2 - 5 }
\end{tabular} & 188 & 12 & 0 \\
\hline \multirow{2}{*}{1 Minute } & \begin{tabular}{c} 
NanoPure water \\
\cline { 2 - 5 }
\end{tabular} & 66 & 28 & 25 \\
\cline { 2 - 5 } & $\begin{array}{c}12.65 \text { mM Glucose } \\
\text { in NanoPure water }\end{array}$ & 84 & 18 & 0 \\
\hline \multirow{2}{*}{20 Minutes } & \begin{tabular}{c} 
NanoPure water \\
\cline { 2 - 5 }
\end{tabular} & 30 & 7 & 0 \\
\hline
\end{tabular}


Only the Control devices actually demonstrated a trend based on the glucose concentration thus the petri wash method was ultimately abandoned. Thus, the focus shifted back to determining the effect of the wash through method. The method was tested using a serial dilution of the stock solution with a concentration of $2.369 \% \mathrm{w} / \mathrm{w}$. After the wash step, the devices were tested using NanoPure water and one NanoPureGlucose solution (12.65 mM). However, all the devices failed to finish again though, like the previous test with this wash method, the sample made it through the polymer layer to the channel below for many of the devices. This result seems to suggest that the wash step is actually dissolving some of the polymer and transporting it through the device. This might explain why the sample for some of these devices is able to make through the polymer layer to the channel below.

Another serial dilution was performed producing another polymer solution with a concentration of $1.186 \% \mathrm{w} / \mathrm{w}$. This solution was tested using the wash through method using the same solutions and the same samples. In this case, two of the acid-washed devices tested with the NanoPure-Glucose solution $(12.65 \mathrm{mM})$ finished this time at around 20 minutes though the device times were separated by about 4 minutes.

Given these results, the hypothesis was that there might still be too much polymer present so another serial dilution was performed producing a $0.595 \% \mathrm{w} / \mathrm{w}$ solution. This solution was tested was using the wash through method with the same solutions and samples being used. The results for these tests are denoted below in Table 33 . 
Table 33: Wash Through Results for $0.595 \%$ w/w Polymer Solution $(n=4)$

\begin{tabular}{|c|c|c|c|c|}
\hline Wash Method & Sample & Avg. t (s) & StDev. (s) & $\%$ Failed \\
\hline \multirow{2}{*}{$\begin{array}{l}\text { NanoPure } \\
\text { water }\end{array}$} & NanoPure water & 1368 & 385 & 50 \\
\hline & $\begin{array}{l}12.65 \mathrm{mM} \text { Glucose } \\
\text { in NanoPure water }\end{array}$ & 922 & 165 & 50 \\
\hline \multirow{2}{*}{$0.005 \mathrm{M} \mathrm{HCl}$} & NanoPure water & 297 & 201 & 0 \\
\hline & $\begin{array}{l}12.65 \mathrm{mM} \text { Glucose } \\
\text { in NanoPure water }\end{array}$ & 127 & 9 & 0 \\
\hline
\end{tabular}

The acid wash results seemed to be promising as there is a clear trend. On the other hand, the large standard deviation for the NanoPure water sample means that the results are not statistically significant. Thus, the acid wash tests were performed again using NanoPure water, 1XPBS, one NanoPure-Glucose solution (12.65 mM), and one 1XPBS-Glucose solution $(13.53 \mathrm{mM})$ to determine if the results were reproducible. In addition, another polymer solution was prepared with a concentration of $0.913 \% \mathrm{w} / \mathrm{w}$ polymer. This solution was tested as well using the acid wash method with NanoPure water and one NanoPure-Glucose solution (12.65 mM). The results are noted in Table 34 . 
Table 34: Acid Wash Method Results $(n=4)$

\begin{tabular}{|c|c|c|c|c|}
\hline Polymer Solution & Sample & Avg. t (s) & StDev. (s) & \% Failed \\
\hline \multirow{5}{*}{$0.595 \% \mathrm{w} / \mathrm{w}$} & NanoPure water & 306 & 73 & 0 \\
\cline { 2 - 5 } & $\begin{array}{c}12.65 \mathrm{mM} \text { Glucose } \\
\text { in NanoPure water }\end{array}$ & 246 & 83 & 0 \\
\cline { 2 - 5 } & 1 XPBS & 21 & 4 & 0 \\
\cline { 2 - 5 } & $\begin{array}{c}13.53 \mathrm{mM} \text { Glucose } \\
\text { in 1XPBS }\end{array}$ & 23 & 5 & 0 \\
\hline \multirow{2}{*}{$0.913 \% \mathrm{w} / \mathrm{w}$} & $\begin{array}{c}\text { NanoPure water } \\
\text { 12.65 mM Glucose } \\
\text { in NanoPure water }\end{array}$ & 249 & 100 & 0 \\
\hline
\end{tabular}

For the $0.595 \% \mathrm{w} / \mathrm{w}$ solution, the results lost the original trend that was seen in the previous test and no longer seem to denote any type of trend when combined with the results for the 1 XPBS samples. The $0.913 \% \mathrm{w} / \mathrm{w}$ solution times seem to change with a change in the glucose concentration, but the results are completely overwhelmed by the large standard deviations for the devices. As a result, no further wash tests were performed using the vertical flow devices.

In addition to the wash tests performed on the vertical flow devices, the wash through method was also tested on the lateral flow bone devices. These devices used the $2.369 \% \mathrm{w} / \mathrm{w}$ solution, were washed using the same solutions, and were tested with the same samples as well to determine if the lateral flow devices might respond differently. The results for this test are noted in Table 35. 
Table 35: Wash Through Testing with Bone Devices $(n=3)$

\begin{tabular}{|c|c|c|c|c|}
\hline Wash Method & Sample & Avg. t (s) & StDev. (s) & \% Failed \\
\hline \multirow{2}{*}{ NanoPure water } & \begin{tabular}{c} 
NanoPure water \\
\cline { 2 - 5 } $0.005 \mathrm{M} \mathrm{HCl}$
\end{tabular} & 124 & 15 & 0 \\
\cline { 2 - 5 } & $\begin{array}{c}12.65 \text { mM Glucose } \\
\text { in NanoPure water }\end{array}$ & 122 & 21 & 0 \\
\cline { 2 - 5 } & $\begin{array}{l}\text { NanoPure water } \\
\text { in NanoPure water }\end{array}$ & 209 & 35 & 0 \\
\hline
\end{tabular}

The results for the lateral devices failed to exhibit any significant trends that would be worthwhile to investigate further. Thus, the wash methods for both the vertical flow and the lateral flow devices ultimately failed to reduce the standard deviations while maintaining the overall trend noted in earlier tests. As a result, the wash preparation methods were abandoned from further testing. The overall research focus shifted as the active hypothesis became that polymeric reactions with the cellulosic matrix of the substrate were interfering with reactions between the polymer assay and glucose. Thus, the remaining tests performed tested using two different substrates, nitrocellulose and printer paper, to fabricate the polymer well panel and modifying the polymer well panel using sodium periodate to oxidize the hydroxyl groups on the cellulose.

The Nitrocellulose and Printer Paper polymer well panels were first tested using the stock polymer solution with NanoPure water and one NanoPure-Glucose solution $(12.65 \mathrm{mM})$ to determine how the polymer would react in the context of a different substrate material. The results for these tests are denoted in Table 36 . 
Table 36: Nitrocellulose and Printer Paper Tests with $4.685 \%$ w/w Solution (n=4)

\begin{tabular}{|c|c|c|c|c|}
\hline Substrate & Sample & Avg. t (s) & StDev. (s) & \% Failed \\
\hline \multirow{3}{*}{ Nitrocellulose } & NanoPure water & 94 & 5 & 0 \\
\cline { 2 - 5 } Printer Paper & $\begin{array}{l}12.65 \text { mM Glucose } \\
\text { in NanoPure water }\end{array}$ & 122 & 31 & 0 \\
\cline { 2 - 5 } & $\begin{array}{l}\text { NanoPure water } \\
\text { in NanoPure water }\end{array}$ & 184 & 97 & 0 \\
\hline
\end{tabular}

Because the samples made it through the polymer so quickly, the tests were performed again using the same samples though this time double the amount $(2 \mu \mathrm{L})$ was deposited in the polymer wells. The results for these tests are denoted below in Table 37.

Table 37: Nitrocellulose and Printer Paper Tests with $2 \mu \mathrm{L}$ of Solution (n=4)

\begin{tabular}{|c|c|c|c|c|}
\hline \multirow{2}{*}{ Substrate } & Sample & Avg. t (s) & StDev. (s) & \% Failed \\
\hline \multirow{2}{*}{ Nitrocellulose } & NanoPure water & 98 & 7 & 0 \\
\cline { 2 - 5 } & $12.65 \mathrm{mM}$ Glucose & 104 & 6 & 0 \\
\hline \multirow{2}{*}{ Printer Paper } & NanoPure water & 367 & 93 & 0 \\
\cline { 2 - 5 } & $12.65 \mathrm{mM}$ Glucose & 403 & N/A & 75 \\
\hline
\end{tabular}

For the devices that failed to finish above, the sample again made it through the polymer layer into the channel below. These results show no difference between the blank (NanoPure water) and the glucose sample. The tests were performed again using the same samples though now with $4 \mu \mathrm{L}$ of the solution deposited. In addition, tests were 
performed using the $3.40 \% \mathrm{w} / \mathrm{w}$ Methanol-NanoPure polymer solution. The results for these tests are denoted below in Table 38.

Table 38: Nitrocellulose and Printer Paper Test Results for Aqueous and Methanol-NanoPure Solutions $(n=4)$

\begin{tabular}{|c|c|c|c|c|c|}
\hline $\begin{array}{l}\text { Polymer } \\
\text { Solution }\end{array}$ & Substrate & Sample & Avg. t (s) & StDev. (s) & $\%$ Failed \\
\hline \multirow{4}{*}{$4.685 \% \mathrm{w} / \mathrm{w}$} & \multirow{2}{*}{ Nitrocellulose } & NanoPure water & 762 & $\mathrm{~N} / \mathrm{A}$ & 75 \\
\hline & & $12.65 \mathrm{mM}$ Glucose & N/A & $\mathrm{N} / \mathrm{A}$ & 100 \\
\hline & \multirow{2}{*}{ Printer Paper } & NanoPure water & 1169 & $\mathrm{~N} / \mathrm{A}$ & 75 \\
\hline & & $12.65 \mathrm{mM}$ Glucose & N/A & $\mathrm{N} / \mathrm{A}$ & 100 \\
\hline \multirow{4}{*}{$3.40 \% \mathrm{w} / \mathrm{w}$} & \multirow{2}{*}{ Nitrocellulose } & NanoPure water & 73 & 12 & 0 \\
\hline & & $12.65 \mathrm{mM}$ Glucose & 60 & 8 & 0 \\
\hline & \multirow{2}{*}{ Printer Paper } & NanoPure water & 1365 & 1109 & 50 \\
\hline & & $12.65 \mathrm{mM}$ Glucose & 1781 & $\mathrm{~N} / \mathrm{A}$ & 75 \\
\hline
\end{tabular}

The Methanol-NanoPure solution results were disappointing with the nitrocellulose devices having no difference between the two samples and the printer paper devices exhibiting large standard deviations for the devices that finished. The $4 \mu \mathrm{L}$ devices, on the other hand, were of interest as they demonstrated how much polymer was necessary to stop most of the devices for these substrate. Thus, the tests were repeated using the same samples and the same polymer solution though with $3 \mu \mathrm{L}$ deposited on the nitrocellulose panels and with $2 \mu \mathrm{L}$ deposited on the Printer Paper panels. The results for these tests are denoted in Table 39. 
Table 39: Nitrocellulose and Printer Paper Results $(n=4)$

\begin{tabular}{|c|c|c|c|c|}
\hline Substrate & Sample & Avg. t (s) & StDev. (s) & \% Failed \\
\hline \multirow{2}{*}{ Nitrocellulose } & NanoPure water & 610 & 14 & 50 \\
\cline { 2 - 5 } & $12.65 \mathrm{mM}$ Glucose & N/A & N/A & 100 \\
\hline \multirow{2}{*}{ Printer Paper } & NanoPure water & 319 & 142 & 0 \\
\cline { 2 - 5 } & $12.65 \mathrm{mM}$ Glucose & N/A & N/A & 100 \\
\hline
\end{tabular}

The nitrocellulose results were quite disappointing for this round of testing. Given the results produced by the nitrocellulose panels, testing using the panels was suspended in favor of investigating the printer paper panels further. The printer paper panels were tested again with $2 \mu \mathrm{L}$ of the $4.685 \% \mathrm{w} / \mathrm{w}$ solution using four different NanoPureGlucose solutions $(6.325 \mathrm{mM}, 3.163 \mathrm{mM}, 1.58 \mathrm{mM}$, and $0.791 \mathrm{mM})$ to try top determine if there was any trend present with a change in the glucose concentration. The results for these tests are denoted below in Table 40.

Table 40: Printer Paper Panel with 4.685 \% w/w Solution (n=4)

\begin{tabular}{|c|c|c|c|}
\hline Sample & Avg. t (s) & StDev. (s) & \% Failed \\
\hline $6.325 \mathrm{mM}$ Glucose & 360 & 151 & 25 \\
\hline $3.163 \mathrm{mM}$ Glucose & 230 & 74 & 50 \\
\hline $1.58 \mathrm{mM}$ Glucose & 377 & 87 & 0 \\
\hline $0.791 \mathrm{mM}$ Glucose & 340 & 53 & 0 \\
\hline
\end{tabular}

These results demonstrate no trend at all between the various glucose concentrations for the printer paper panels. As a result, the research into this was halted 
in favor of research on cellulose modification research. The modified polymer panel was tested using the $4.685 \% \mathrm{w} / \mathrm{w}$ solution with NanoPure water and one NanoPure-Glucose solution $(12.65 \mathrm{mM})$. The results for these tests are denoted in Table 41 .

Table 41: Modified Panel with $4.685 \%$ w/w Solution $(n=4)$

\begin{tabular}{|c|c|c|c|}
\hline Sample & Avg. t (s) & StDev. (s) & \% Failed \\
\hline NanoPure water & 484 & 70 & 0 \\
\hline 12.65 mM Glucose & 554 & 82 & 0 \\
\hline
\end{tabular}

While these results did demonstrate a trend, they were not different in any way from the results obtained previously with the unmodified chromatography paper. This seems to suggest either the cellulose does not affect the reaction between the glucose and the polymer or that the cellulose was not oxidized enough by the sodium periodate solution to effectively remove any reaction between the polymer and the cellulose. Upon further analysis of the results obtained, the most likely answer is that the polymer is simply not sensitive enough to detect differences in the glucose concentration within a range that could be effectively utilized on a microPAD for POC diagnostics in either an RLS or a clinical environment.

One possible explanation for the large amount of variability present with the 3D devices was the method by which the devices were constructed after fabrication and deposition of the polymer solution. The bolts for the devices were handtightened to the maximum torque before the devices were used. However, informal studies have shown that the amount of torque applied to the bolts can influence the device times. No formal studies have yet been undertaken to determine how large of an effect is present. Thus, the 
construction method for the 3D devices may have introduced an amount of subjective variability.

In addition, the effects of either the molecular weight or the PDI of the polymer assay were never examined closely due to both time and material constraints related to the synthesis of the polymer assay. Both areas of research could drastically change the behavior of the assay within the context of both the $2 \mathrm{D}$ and the $3 \mathrm{D}$ devices. For instance, an increase in the molecular weight for the polymer would increase the overall device times but could, in theory, produce an assay with a greater sensitivity to smaller changes in the glucose assay. That is, more minute concentration differences would be easier to detect. On the other hand, a smaller molecular weight polymer would allow the devices to finish faster overall making them more effective for clinical diagnostic applications. However, the polymer would not be as sensitive now to more minor differences in glucose concentrations.

The effect of the PDI on the PBBA reactivity would also be an interesting area of research to explore as it might allow a better understanding of the reactions between the polymer and the paper to be developed. For instance, a low PDI polymer assay should, in theory, have less variability between devices as the sample is encountering polymer chains that are very close together in terms of molecular weight. However, a low PDI polymer assay might not fill the cellulosic pores of the substrate as effectively due to a lower packing density and might have a lower effective range for glucose limiting the possible range of applications. 
On the other hand, a high PDI polymer assay could possibly more effectively block the cellulosic pores of the substrate due to the theoretically higher packing density and improve the effective range of the assay. A higher effective range could increase the range of possible applications for the assay and increase the viability of using the assay within microPADs. However, a higher PDI polymer assay could increase the amount of variability present in the devices as the sample is now reacting with a broader range of polymer chain sizes. Each of these different molecular weights will flow through the device at a different flow rate.

In addition to the effects of molecular weight and PDI, the chemical structure of the polymer could also be investigated as well. The polymer samples synthesized in collaboration with the Sumerlin group at the University of Florida were intended to be used to determine the effect of the monomeric unit on the PBBA reactivity. However, these samples could not be obtained in time to be tested alongside the initial PAPBA sample provided.

Finally, one possible explanation for the variability present for the final aqueous solutions could be present in the dilution method. The final aqueous polymer dilutions were all prepared using NanoPure water whereas the initial aqueous solutions were all prepared using $0.1 \mathrm{M} \mathrm{NaOH}$ instead. The result is that the dilutions for the final aqueous solutions experienced both a drop in the polymer concentration as well as in the $\mathrm{pH}$ of the solution. As a result, the ionic strength for the solutions decreased as well as the dilutions were prepared. The decrease in the ionic strength might have made the polymer more susceptible to localized minor differences in the glucose samples introducing an amount of variability to the devices. On the other hand, the decrease in the ionic strength of the 
polymer could have destabilized the polymer in the solution. This could, in theory, make it easier for the polymer to precipitate out of the solution after it deposited on the device and allow a more effective, temporary barrier to be formed. 


\section{CONCLUSIONS}

Polymer Bound Boronic Acid, a glucose and $\mathrm{pH}$ sensitive polymer, was investigated for use in developing a paper-based chronometric glucose assay. One common theme for the results from the glucose assays was that any glucose dependent trend for the assay noted was always present alongside large device standard deviations. The signal either appeared to be a function of glucose concentration, though with large standard deviations rendering the results statistically insignificant, or the signal had low standard deviations, though with no apparent relationship to the concentration of glucose in the sample. Upon further analysis of the results, the overall hypothesis is that the polymer is simply not sensitive enough to detect differences in the glucose concentration within a range that would be practical for either POC or clinical diagnostic applications.

Possible future directions for this research could focus on examining the relationship between the polymer and the device. In particular, for this sample, the effect of the polymer PDI and molecular weight could be studied further to reduce the large standard deviations received from the devices. In addition, the monomeric composition could be varied to determine what effect the polymer backbone has on the reaction between the polymer particles and the glucose within the substrate. Finally, a copolymer such as a block- or alternating-copolymer could be investigated as well to either act as a spacer in the polymer chains or to modify the reactivity of the assay with both other polymer particles as well as with glucose containing samples. 


\section{REFERENCES}

(1) Cambre, J. N.; Roy, D.; Sumerlin, B. S. J. Polym. Sci. Part A Polym. Chem. 2012, 50 (16), 3373-3382.

(2) Roy, D.; Sumerlin, B. S. ACS Macro Lett. 2012, 1 (5), 529-532.

(3) Cate, D. M.; Adkins, J. A.; Mettakoonpitak, J.; Henry, C. S. Anal. Chem. 2014, 87 (Fundamental and Applied Reviews in Analytical Chemistry 2015), 19-41.

(4) Yetisen, A. K.; Akram, M. S.; Lowe, C. R. Lab Chip 2013, 13 (12), 2210-2251.

(5) Lu, Y.; Shi, W.; Jiang, L.; Qin, J.; Lin, B. Electrophoresis 2009, 30 (9), 14971500.

(6) Carrilho, E.; Martinez, A. W.; Whitesides, G. M. Anal. Chem. 2009, 81 (16), 1-5.

(7) Lewis, G. G.; Robbins, J. S.; Phillips, S. T. Anal. Chem. 2013, 85 (21), 1043210439.

(8) Pappin, B.; Kiefel, M. J.; Houston, T. A. In Carbohydrates - Comprehensive Studies on Glycobiology and Glycotechnology; 2012; pp 37-54.

(9) Odian, G. Principles of Polymerization, 4th ed.; John Wiley \& Sons, Inc.: Staten Island, New York, 2012.

(10) Butt, H.-J.; Graf, K.; Kappl, M. Physics and Chemistry of Interfaces; 2013.

(11) Kim, U.-J.; Kuga, S.; Wada, M.; Okano, T.; Kondo, T. Biomacromolecules 2000, 1 (3), 488-492.

(12) Coleman, M. C.; Painter, P. C. Fundamentals of Polymer Science: An Introductory Text; CRC Press, 1998. 
(13) Lu, Y.; Shi, W.; Qin, J.; Lin, B. Anal. Chem. 2010, 82 (1), 329-335.

(14) Co, C. C.; Cotts, P.; Burauer, S.; De Vries, R.; Kaler, E. W. Macromolecules 2001, 34 (10), 3245-3254.

(15) Brooks, W. L. A.; Sumerlin, B. S. Chem. Rev. 2016, 116 (3), 1375-1397.

(16) Hoglund, E. Production of Dialdehyde Cellulose and Periodate Regeneration: Towards feasible oxidation processes, 2015.

(17) Ma, S.; Tang, Y.; Liu, J.; Wu, J. Talanta 2014, 120, 135-140.

(18) Martinez, A. W.; Phillips, S. T.; Wiley, B. J.; Gupta, M.; George, M. NIH Public Access 2011, 8 (12), 2146-2150.

(19) OuYang, L.; Wang, C.; Du, F.; Zheng, T.; Liang, H. RSC Adv. 2014, 4 (3), 1093.

(20) Garcia, P. de T.; Cardoso, T. M. G.; Garcia, C. D.; Carrilho, E.; Coltro, W. K. T. RSC Adv. 2014, 4 (71), 37637-37644.

(21) Zhang, Y. et al. Anal. Chem. 2014, 86, 2005-2012.

(22) Abe, K.; Suzuki, K.; Citterio, D. Anal. Chem. 2008, 80 (18), 6928-6934.

(23) Maejima, K.; Tomikawa, S.; Suzuki, K.; Citterio, D. RSC Adv. 2013, 3 (24), 9258.

(24) Yamada, K.; Takaki, S.; Komuro, N.; Suzuki, K.; Citterio, D. Analyst 2014, 139 (7), 1637-1643.

(25) Li, X.; Tian, J.; Garnier, G.; Shen, W. Colloids Surfaces B Biointerfaces 2010, 76, 564-570.

(26) Wang, J. et al. Lab Chip 2014, 14 (4), 691-695.

(27) Olkkonen, J.; Lehtinen, K.; Erho, T. Anal. Chem. 2010, 82 (24), 10246-10250.

(28) Määttänen, A. et al. Sensors Actuators, B Chem. 2011, 160 (1), 1404-1412. 
(29) Deiss, F.; Matochko, W. L.; Govindasamy, N.; Lin, E. Y.; Derda, R. Angew. Chemie - Int. Ed. 2014, 53 (25), 6374-6377.

(30) Songjaroen, T.; Dungchai, W.; Chailapakul, O.; Laiwattanapaisal, W. Talanta 2011, 85 (5), 2587-2593.

(31) Noiphung, J. et al. Anal. Chim. Acta 2013, 788, 39-45.

(32) Fenton, E. M.; Mascarenas, M. R.; López, G. P.; Sibbett, S. S. ACS Appl. Mater. Interfaces 2009, 1 (1), 124-129.

(33) Fu, E.; Kauffman, P.; Lutz, B.; Yager, P. Sensors Actuators, B Chem. 2010, 149 (1), 325-328.

(34) Renault, C.; Li, X.; Fosdick, S. E.; Crooks, R. M. Anal. Chem. 2013, 85, 79767979.

(35) Cassano, C. L.; Fan, Z. H. Microfluid. Nanofluidics 2013, 15 (2), 173-181.

(36) Giokas, D. L.; Tsogas, G. Z.; Vlessidis, A. G. Anal. Chem. 2014, 86 (13), 6202 6207.

(37) Nie, J. et al. Analyst 2013, 138 (2), 671-676.

(38) Evans, E.; Gabriel, E. F. M.; Coltro, W. K. T.; Garcia, C. D. Analyst 2014, 139 (9), $2127-2132$.

(39) Fridley, G. E.; Le, H.; Yager, P. Anal. Chem. 2014, 86 (13), 6447-6453.

(40) Thuo, M. M. et al. Chem. Mater. 2014, 26 (14), 4230-4237.

(41) Mitchell, H. T. et al. Lab Chip 2015, 15, 17-20.

(42) Martinez, A. W.; Phillips, S. T.; Butte, M. J.; Whitesides, G. M. Angew. Chemie Int. Ed. 2007, 46 (8), 1318-1320.

(43) Roy, D.; Sumerlin, B. S. ACS Macro Lett. 2012, 1 (5), 529-532. 